\title{
ANALYSIS AND APPLICATIONS OF HOLOMORPHIC FUNCTIONS IN HIGHER DIMENSIONS
}

\author{
R. Z. YEH
}

\begin{abstract}
Holomorphic functions in $R^{n}$ are defined to generalize those in $R^{2}$. A Taylor formula and a Cauchy integral formula are presented. An application of the Taylor formula to the kernel of the Cauchy integral formula results in Taylor series expansions of holomorphic functions. Real harmonic functions are expanded in series of homogeneous harmonic polynomials.
\end{abstract}

\section{INTRODUCTION}

One of the main results in complex analysis in $R^{2}$ is that a function holomorphic in the sense of Cauchy-Riemann is analytic in the sense of Taylor. We recall the celebrated Cauchy integral formula leading to Taylor series expansions of holomorphic functions through geometric series expansion of its kernel. In $R^{n}$ the situations are similar, but not as straightforward. First we construct a Cauchy integral formula, then we find a Taylor formula to expand its kernel, but the remainder term turns out quite difficult to estimate. Nevertheless, obstacles are overcome, and we have analyticity of holomorphic functions in $R^{n}$, a special case of which is the classical result in $R^{2}$. Taylor series expansions of holomorphic functions give rise to expansions of real harmonic functions in series of homogeneous harmonic polynomials, and as a consequence we have an algorithm for finding complete sets of independent homogeneous harmonic polynomials. In $R^{2}$ it is a simple matter of taking the real and the imaginary components of powers of the complex variable $z$. In $R^{n}$ it is a more elaborate process of first computing the symmetric powers of hypercomplex variables $z_{1}, z_{2}, \ldots, z_{n-1}$, and then selecting, besides the real components, some of the imaginary components. Surprisingly, while there are $n-1$ imaginary components in each symmetric power, most of them are repeated by components of other symmetric powers, and we need only choose the first and ignore the rest. Thus, the polynomial expansions of harmonic functions in $R^{2}$ and $R^{n}$ are practically of the same form, and we have in fact the best possible generalization of results in $R^{2}$ to $R^{n}$.

Received by the editors April 5, 1993.

1991 Mathematics Subject Classification. Primary 35C10, 31B05, 30G35.

Key words and phrases. Hypercomplex numbers, holomorphic functions, Cauchy-Riemann equations, symmetric powers, Stieltjes line integrals, Taylor formula, Cauchy integral formula, divergence theorem, Leibniz's rule, power series, harmonic functions, Poisson equation, Laplace equation, polynomial expansions. 


\section{HYPERCOMPLEX NUMBERS}

Imitating the complex number system $C_{1}$ for the plane $R^{2}$, we consider a hypercomplex number system $C_{n-1}$ for the space $R^{n}$ by creating $n-1$ imaginary units $e_{1}, e_{2}, \ldots, e_{n-1}$ subject to multiplication rules:

$$
\begin{gathered}
e_{i} e_{j}=-e_{j} e_{i} \text { for } i \neq j, \\
e_{1}^{2}=e_{2}^{2}=\cdots=e_{n-1}^{2}=-1 .
\end{gathered}
$$

A hypercomplex number is expressible in the component form

$$
p=b_{0}+e_{1} b_{1}+\cdots+e_{n-1} b_{n-1}+e_{12} b_{12}+e_{13} b_{13}+\cdots,
$$

where $e_{12}:=e_{1} e_{2}$ and $e_{13}:=e_{1} e_{3}$ are the first two of the many hyperimaginary units arising from multiplications of any number of imaginary units. More formally,

$$
p=\sum_{\lambda} e_{\lambda} b_{\lambda},
$$

where each component $b_{\lambda}$ is real and each $\lambda$ such as $\langle 1,2\rangle$ or $\langle 1,3\rangle$ is any one of the $2^{n-1}$ order-preserving subsets, including the empty set $\varnothing$, of the ordered set $\langle 1,2, \ldots, n-1\rangle$. Needless to say, we identify $e_{\varnothing}$ with 1 and $b_{\varnothing}$ with $b_{0}$. Although hypercomplex numbers can be cumbersome, often we need only the polycomplex numbers. These are hypercomplex numbers whose hyperimaginary components are zero, and we shall express such a number in the form

$$
p=e_{1} b_{1}+\cdots+e_{n-1} b_{n-1}+b_{n},
$$

where we prefer to label the real component as $b_{n}$. The conjugate $\bar{p}$ of $p$ is obtained from $p$ by replacing each and every $e_{i}$ found in $p$ by $-e_{i}$. Thus,

$$
\bar{p}=\sum_{\lambda} \bar{e}_{\lambda} b_{\lambda} \text { and } \bar{e}_{\lambda}=(-1)^{|\lambda|} e_{\lambda},
$$

where $|\lambda|$ is the cardinality of $\lambda$. In particular, $\bar{e}_{i}=-e_{i}$, and it is not difficult to see

$$
\overline{p q}=\bar{p} \bar{q},
$$

and that if $p$ is polycomplex, then

$$
p \bar{p}=\bar{p} p=|p|^{2},
$$

where $|p|$ is the absolute value of $p$, defined for all hypercomplex $p$ as the square root of the sum of all the components squared.

In Hile-Lounesto [6] the smallest number $\kappa_{n-1}$ is determined such that

$$
|p q| \leq \kappa_{n-1}|p||q|
$$

for all $p, q$ in $C_{n-1}$. However, for polycomplex $p$ and $q$, this inequality can be replaced by a simple equality. 
Lemma 1.1. If $p$ and $q$ are both polycomplex, then

$$
|p q|=|p||q| \text {. }
$$

Proof. Let $p=\sum_{i=1}^{n} e_{i} b_{i}$ and $q=\sum_{j=1}^{n} e_{j} a_{j}$, where $e_{n}$ is taken to be 1 , then with $e_{i} \bar{e}_{i}=1$ for all $i$,

$$
\begin{aligned}
p \bar{q} & =\left(\sum_{i} e_{i} b_{i}\right)\left(\sum_{j} \bar{e}_{j} a_{j}\right)=\sum_{i, j} e_{i} \bar{e}_{j} b_{i} a_{j} \\
& =\left(\sum_{i=j}+\sum_{i<j}+\sum_{i>j}\right) e_{i} \bar{e}_{j} b_{i} a_{j}=\sum_{i} b_{i} a_{i}+\sum_{i<j} e_{i} \bar{e}_{j}\left(b_{i} a_{j}-b_{j} a_{i}\right) .
\end{aligned}
$$

Hence,

$$
|p \bar{q}|^{2}=\left(\sum_{i} b_{i} a_{i}\right)^{2}+\sum_{i<j}\left(b_{i} a_{j}-b_{j} a_{i}\right)^{2}=\left(\sum_{i} b_{i}^{2}\right)\left(\sum_{j} a_{j}^{2}\right)=|p|^{2}|\bar{q}|^{2} .
$$

Replacing $\bar{q}$ by $q$, we obtain $|p q|^{2}=|p|^{2}|q|^{2}$.

\section{HoLOMORPHIC FUnCTIONS}

We consider only hypercomplex functions $f(\mathbf{x})=\sum_{\lambda} e_{\lambda} v_{\lambda}(\mathbf{x})$ whose components are smooth, that is, partial derivatives of $\nu_{\lambda}$ up to a certain order are all continuous in some domain $\Omega$ in $R^{n}$. Occasionally we will focus on smooth polycomplex functions $f(\mathbf{x})=\sum_{i=1}^{n} e_{i} v_{i}(\mathbf{x})$ where $e_{n}:=1$. For smooth hypercomplex $f$ we consider the polycomplex differential operator $\partial$ defined by

$$
\partial:=e_{1} \partial_{1}+e_{2} \partial_{2}+\cdots+e_{n-1} \partial_{n-1}+\partial_{n},
$$

where $\left(\partial_{1}, \partial_{2}, \ldots, \partial_{n}\right)=\boldsymbol{\partial}$ is the usual gradient operator, more commonly denoted as $\nabla$, and

$$
\begin{gathered}
\partial f=\left(\sum_{i=1}^{n} e_{i} \partial_{i}\right)\left(\sum_{\lambda} e_{\lambda} v_{\lambda}\right)=\sum_{i=1}^{n} \sum_{\lambda}\left(e_{i} e_{\lambda}\right)\left(\partial_{i} v_{\lambda}\right), \\
f \partial=\left(\sum_{\lambda} e_{\lambda} v_{\lambda}\right)\left(\sum_{i=1}^{n} e_{i} \partial_{i}\right)=\sum_{\lambda} \sum_{i=1}^{n}\left(e_{\lambda} e_{i}\right)\left(\partial_{i} v_{\lambda}\right) .
\end{gathered}
$$

We say that $f$ is holomorphic if $\bar{\partial} f=0$, and retroholomorphic if $f \bar{\partial}=0$, where the operator $\bar{\partial}:=\bar{e}_{1} \partial_{1}+\bar{e}_{2} \partial_{2}+\cdots+\bar{e}_{n-1} \partial_{n-1}+\partial_{n}$ is the conjugate of $\partial$.

Theorem 2.1 (equations of holomorphy). A polycomplex function $f=e_{1} v_{1}$ $+\cdots+e_{n-1} v_{n-1}+v_{n}$ is holomorphic in a domain $\Omega$ in $R^{n}$ if and only if its components satisfy in $\Omega$

$$
\begin{gathered}
\partial_{1} v_{1}+\cdots+\partial_{n} v_{n}=0, \\
\partial_{i} v_{j}=\partial_{j} v_{i} \quad \text { for } 1 \leq i \neq j \leq n .
\end{gathered}
$$

Proof. Substituting the component forms of $\bar{\partial}$ and $f$ in $-\bar{\partial} f=0$, we have

$$
\left(e_{1} \partial_{1}+\cdots+e_{n-1} \partial_{n-1}-\partial_{n}\right)\left(e_{1} v_{1}+\cdots+e_{n-1} v_{n-1}+v_{n}\right)=0 .
$$


Expanding and setting each component to zero, we obtain (2.1) and (2.2). Conversely, if (2.1) and (2.2) are satisfied, then (2.3) holds and $\bar{\partial} f=0$.

In particular, if $n=2$, we have $f=e_{1} v_{1}+v_{2}$, and

$$
\partial_{1} v_{1}+\partial_{2} v_{2}=0, \quad \partial_{1} v_{2}=\partial_{2} v_{1} \text {. }
$$

Now, in complex analysis, $e_{1}$ is usually written as $i, \nu_{1}$ is written as $\nu$, and $\nu_{2}$ is written as $u$, so that $f=i \nu+u$, and if partial differentiations are denoted by subscripts, then we have

$$
\begin{gathered}
v_{x}+u_{y}=0, \\
u_{x}=v_{y} .
\end{gathered}
$$

Equations (2.4) and (2.5) are the well-known Cauchy-Riemann equations in $R^{2}$.

Theorem 2.2. A polycomplex function $f=e_{1} v_{1}+\cdots+e_{n-1} v_{n-1}+v_{n}$ is retroholomorphic if and only if it is holomorphic.

Proof. It is easy to check that for a polycomplex $f$ the respective components of $\bar{\partial} f$ and $f \bar{\partial}$ are either equal or negative of each other. Hence, if $f$ is polycomplex, $\bar{\partial} f$ and $f \bar{\partial}$ always vanish simultaneously.

There are $n-1$ basic holomorphic functions in $R^{n}$, each corresponding to one imaginary unit. They are given by

$$
z_{i}(\mathbf{x})=x_{i}+e_{i} x_{n} \quad \text { for } 1 \leq i \leq n-1
$$

with $\mathbf{x}=\left(x_{1}, x_{2}, \ldots, x_{n}\right)$. For $n=2$, we have simply $z_{1}=x_{1}+e_{1} x_{2}=x+i y$. Clearly,

$$
\bar{\partial} z_{i}=\left(\bar{e}_{i} \partial_{i}\right) x_{i}+\partial_{n}\left(e_{i} x_{n}\right)=\bar{e}_{i}+e_{i}=0 .
$$

In trying to find more holomorphic functions, we note that while linear combinations of $z_{i}$ are holomorphic their products may not be. For example, $z_{1} z_{2}$ is not holomorphic since

$$
\begin{aligned}
\bar{\partial}\left(z_{1} z_{2}\right) & =\bar{\partial}\left[\left(x_{1}+e_{1} x_{n}\right)\left(x_{2}+e_{2} x_{n}\right)\right] \\
& =\bar{\partial}\left[x_{1} x_{2}+e_{1}\left(x_{2} x_{n}\right)+e_{2}\left(x_{1} x_{n}\right)+e_{1} e_{2} x_{n}^{2}\right] \\
& =\left(\bar{e}_{1} x_{2}+\bar{e}_{2} x_{1}\right)+\left(\bar{e}_{2} e_{1} x_{n}+e_{1} x_{2}\right)+\left(\bar{e}_{1} e_{2} x_{n}+e_{2} x_{1}\right)+e_{1} e_{2}\left(2 x_{n}\right) \\
& =\left[e_{1}\left(-x_{2}+x_{2}\right)+e_{2}\left(-x_{1}+x_{1}\right)+e_{1} e_{2}\left(x_{n}-x_{n}\right)\right]+e_{1} e_{2}\left(2 x_{n}\right) \\
& =e_{1} e_{2}\left(2 x_{n}\right) .
\end{aligned}
$$

However, we can avoid this difficulty by considering instead $z_{1} z_{2}+z_{2} z_{1}$, then

$$
\bar{\partial}\left(z_{1} z_{2}+z_{2} z_{1}\right)=e_{1} e_{2}\left(2 x_{n}\right)+e_{2} e_{1}\left(2 x_{n}\right)=0 .
$$

Likewise, while $z_{1}^{2} z_{2}$ is not holomorphic, $z_{1}^{2} z_{2}+z_{1} z_{2} z_{1}+z_{2} z_{1}^{2}$ turns out to be. We therefore propose for $Z=\left(z_{1}, z_{2}, \ldots, z_{n-1}\right)$ and each multi-index $\beta=\left(\beta_{1}, \beta_{2}, \ldots, \beta_{n-1}\right)$ the symmetric power $Z^{\beta}$ defined as the sum of all possible $z_{i}$ products each of which contains $z_{i}$ factor exactly $\beta_{i}$ times. For example, with $n=3$, we have

$$
\begin{aligned}
& \left(z_{1}, z_{2}\right)^{(1,1)}=z_{1} z_{2}+z_{2} z_{1}, \\
& \left(z_{1}, z_{2}\right)^{(2,1)}=z_{1}^{2} z_{2}+z_{1} z_{2} z_{1}+z_{2} z_{1}^{2} .
\end{aligned}
$$


Now, in the last equation, if we note further

$$
\left(z_{1}, z_{2}\right)^{(2,1)}=z_{1}\left(z_{1} z_{2}+z_{2} z_{1}\right)+z_{2}\left(z_{1}^{2}\right)=z_{1}\left(z_{1}, z_{2}\right)^{(1,1)}+z_{2}\left(z_{1}, z_{2}\right)^{(2,0)} \text {, }
$$

then we may correctly infer

$$
\left(z_{1}, z_{2}\right)^{\left(\beta_{1}, \beta_{2}\right)}=z_{1}\left(z_{1}, z_{2}\right)^{\left(\beta_{1}-1, \beta_{2}\right)}+z_{2}\left(z_{1}, z_{2}\right)^{\left(\beta_{1}, \beta_{2}-1\right)},
$$

and more generally,

$$
Z^{\beta}=z_{1} Z^{\beta-e^{1}}+z_{2} Z^{\beta-e^{2}}+\cdots+z_{n-1} Z^{\beta-e^{n-1}},
$$

where each $e^{i}$ is the unit multi-index $(0, \ldots, 0,1,0, \ldots 0)$ with 1 occurring only in the $i$ th position. However, rather than proving (2.7) we will use it to define symmetric powers.

\section{SyMmetric POWERS}

We begin with a formal definition of symmetric powers $Z^{\beta}$. Let $Z=$ $\left(z_{1}, z_{2}, \ldots, z_{n-1}\right)$ where $z_{i}=x_{i}+e_{i} x_{n}$, and $\beta=\left(\beta_{1}, \beta_{2}, \ldots, \beta_{n-1}\right)$ where $\beta_{i}$ are integers, then $Z^{\beta}$ is a function in $R^{n}$ defined inductively as follows: If any integer $\beta_{i}$ is negative, we set $Z^{\beta}=0$. If $\beta=(0,0, \ldots, 0)$, we set $Z^{\beta}=1$. And if $|\beta|:=\beta_{1}+\beta_{2}+\cdots+\beta_{n-1} \geq 1$, we set

$$
Z^{\beta}=z_{1} Z^{\beta-e^{1}}+z_{2} Z^{\beta-e^{2}}+\cdots+z_{n-1} Z^{\beta-e^{n-1}}
$$

where $e^{i}=(0, \ldots, 0,1,0, \ldots, 0)$ with 1 occurring only in the $i$ th position. Thus, in $R^{3}$ we have

$$
\begin{aligned}
& \left(z_{1}, z_{2}\right)^{(1,0)}=z_{1}\left(z_{1}, z_{2}\right)^{(0,0)}+z_{2}\left(z_{1}, z_{2}\right)^{(1,-1)}=z_{1}, \\
& \left(z_{1}, z_{2}\right)^{(0,1)}=z_{1}\left(z_{1}, z_{2}\right)^{(-1,1)}+z_{2}\left(z_{1}, z_{2}\right)^{(0,0)}=z_{2}, \\
& \left(z_{1}, z_{2}\right)^{(2,0)}=z_{1}\left(z_{1}, z_{2}\right)^{(1,0)}+z_{2}\left(z_{1}, z_{2}\right)^{(2,-1)}=z_{1}^{2} \\
& \left(z_{1}, z_{2}\right)^{(1,1)}=z_{1}\left(z_{1}, z_{2}\right)^{(0,1)}+z_{2}\left(z_{1}, z_{2}\right)^{(1,0)}=z_{1} z_{2}+z_{2} z_{1}, \\
& \left(z_{1}, z_{2}\right)^{(0,2)}=z_{1}\left(z_{1}, z_{2}\right)^{(-1,2)}+z_{2}\left(z_{1}, z_{2}\right)^{(0,1)}=z_{2}^{2}
\end{aligned}
$$

An alternative formula which we can deduce from (3.1) by induction on $m=|\beta|$ is

$$
Z^{\beta}=Z^{\beta-e^{1}} z_{1}+Z^{\beta-e^{2}} z_{2}+\cdots+Z^{\beta-e^{n-1}} z_{n-1} .
$$

Using either (3.1) or (3.2) we can demonstrate after some calculations (and after rewriting $x_{1}, x_{2}, x_{3}$ as $\left.x, y, z\right)$ :

$$
\begin{aligned}
& \left(z_{1}, z_{2}\right)^{(1,0)}=x+e_{1} z, \\
& \left(z_{1}, z_{2}\right)^{(0,1)}=y+e_{2} z, \\
& \left(z_{1}, z_{2}\right)^{(2,0)}=\left(x^{2}-z^{2}\right)+e_{1}(2 x z), \\
& \left(z_{1}, z_{2}\right)^{(1,1)}=(2 x y)+e_{1}(2 y z)+e_{2}(2 x z), \\
& \left(z_{1}, z_{2}\right)^{(0,2)}=\left(y^{2}-z^{2}\right)+e_{2}(2 y z), \\
& \left(z_{1}, z_{2}\right)^{(3,0)}=\left(x^{3}-3 x z^{2}\right)+e_{1}\left(3 x^{2} z-z^{3}\right), \\
& \left(z_{1}, z_{2}\right)^{(2,1)}=\left(3 x^{2} y-3 y z^{2}\right)+e_{1}(6 x y z)+e_{2}\left(3 x^{2} z-z^{3}\right), \\
& \left(z_{1}, z_{2}\right)^{(1,2)}=\left(3 x y^{2}-3 x z^{2}\right)+e_{1}\left(3 y^{2} z-z^{3}\right)+e_{2}(6 x y z), \\
& \left(z_{1}, z_{2}\right)^{(0,3)}=\left(y^{3}-3 y z^{2}\right)+e_{2}\left(3 y^{2} z-z^{3}\right) .
\end{aligned}
$$


Lemma 3.1. The components of symmetric powers satisfy

$$
\begin{gathered}
\operatorname{comp}_{i} Z^{\beta+e^{i}}=\operatorname{comp}_{j} Z^{\beta+e^{j}} \text { for } 1 \leq i, j \leq n-1, \\
\operatorname{comp}_{\lambda} Z^{\gamma}=0 \text { for all }|\lambda|>1,
\end{gathered}
$$

where $|\lambda|$ denotes the cardinality of $\lambda$.

Proof. We prove the lemma by a simultaneous induction on $|\beta|$ and $|\gamma|$. For $|\beta|=0$ and $|\gamma|=1,(3.3)$ and (3.4) are easily checked. Therefore, we assume (3.3) for $|\beta| \leq k-1$ and (3.4) for $|\gamma| \leq k, k \geq 1$ as induction hypotheses and show (3.3) for $|\beta|=k$ and (3.4) for $|\gamma|=k+1$.

First, for $|\beta|=k$ in (3.3) we use (3.1) to expand $Z^{\beta+e^{i}}=\sum_{q=1}^{n-1} z_{q} Z^{\beta+e^{i}-e^{q}}$, then, by the induction hypothesis on $|\gamma| \leq k$ in (3.4), $Z^{\beta+e^{i}-e^{q}}$ are polycomplex since $\left|\beta+e^{i}-e^{q}\right|=k$, so

$$
\operatorname{comp}_{i} Z^{\beta+e^{i}}=\sum_{q=1}^{n-1} x_{q} \operatorname{comp}_{i} Z^{\beta+e^{i}-e^{q}}+x_{n} \operatorname{Re} Z^{\beta+e^{i}-e^{i}} .
$$

On the other hand, likewise

$$
\operatorname{comp}_{j} Z^{\beta+e^{j}}=\sum_{q=1}^{n-1} x_{q} \operatorname{comp}_{j} Z^{\beta+e^{j}-e^{q}}+x_{n} \operatorname{Re} Z^{\beta+e^{j}-e^{j}}
$$

But, in view of the induction hypothesis on $|\beta| \leq k-1$ in (3.3), we have

$$
x_{q} \operatorname{comp}_{i} Z^{\left(\beta-e^{q}\right)+e^{i}}=x_{q} \operatorname{comp}_{j} Z^{\left(\beta-e^{q}\right)+e^{j}} .
$$

Hence,

$$
\operatorname{comp}_{i} Z^{\beta+e^{i}}=\operatorname{comp}_{j} Z^{\beta+e^{j}}
$$

and (3.3) is confirmed for $|\beta|=k$.

Next, for $|\gamma|=k+1$ in (3.4) we again use (3.1) to expand

$$
Z^{\gamma}=\sum_{i=1}^{n-1} z_{i} Z^{\gamma-e^{i}}
$$

Clearly, $\operatorname{comp}_{\lambda} Z^{\gamma}=0$ for $|\lambda|>2$ since $\operatorname{comp}_{\lambda} Z^{\gamma-e^{i}}=0$ for $|\lambda|>1$ by the induction hypothesis on $|\gamma| \leq k$. It therefore remains to show $\operatorname{comp}_{\lambda} Z^{\gamma}=0$ for $|\lambda|=2$. Now suppose $e_{\lambda}=e_{i} e_{j}$, then since only the $i$ th and the $j$ th terms in (3.5) can possibly produce $e_{\lambda}$, we have

$$
\begin{aligned}
\operatorname{comp}_{\lambda} Z^{\gamma} & =\operatorname{comp}_{\lambda}\left(z_{i} Z^{\gamma-e^{i}}\right)+\operatorname{comp}_{\lambda}\left(z_{j} Z^{\gamma-e^{j}}\right) \\
& =\operatorname{comp}_{\lambda}\left(e_{i} e_{j} x_{n} \operatorname{comp}_{j} Z^{\gamma-e^{i}}\right)+\operatorname{comp}_{\lambda}\left(e_{j} e_{i} x_{n} \operatorname{comp}_{i} Z^{\gamma-e^{j}}\right) \\
& =x_{n} \operatorname{comp}_{j} Z^{\gamma-e^{i}}-x_{n} \operatorname{comp}_{i} Z^{\gamma-e^{j}}
\end{aligned}
$$

But,

$$
\operatorname{comp}_{i} Z^{\gamma-e^{j}}=\operatorname{comp}_{j} Z^{\gamma-e^{i}} \quad(\text { by (3.3) for }|\beta| \leq k-1) \text {. }
$$

So, $\operatorname{comp}_{\lambda} Z^{\gamma}=0$ for $|\lambda|=2$, and (3.4) is confirmed for $|\gamma|=k+1$. 
Lemma 3.2. Every symmetric power $Z^{\beta}$ is a polycomplex holomorphic function in $R^{n}$.

Proof. First $Z^{\beta}$ is polycomplex by (3.4) of the preceding lemma. As for holomorphy of $Z^{\beta}$, we check $\bar{\partial} Z^{\beta}=0$ easily for $|\beta|=1$. So assume as the induction hypothesis $\bar{\partial} Z^{\beta}=0$ for $|\beta| \leq k, k \geq 1$. Now for $|\beta|=k+1$ we first expand $Z^{\beta}$ by (3.2),

$$
Z^{\beta}=\sum_{i=1}^{n-1} Z^{\beta-e^{i}} z_{i}
$$

then $\left|\beta-e^{i}\right|=k$, and $\bar{\partial} Z^{\beta-e^{i}}=0$ by the induction hypothesis. Applying $-\bar{\partial}$ to $Z^{\beta}$ instead of $\bar{\partial}$ for convenience, and noting that each component of $\bar{\partial}$ operates by the product formula of differentiation, we obtain

$$
\begin{aligned}
& (-\bar{\partial}) Z^{\beta}=\left(\sum_{j=1}^{n-1} e_{j} \partial_{j}-\partial_{n}\right)\left(\sum_{i=1}^{n-1} Z^{\beta-e^{i}} z_{i}\right) \\
& =\sum_{i=1}^{n-1}\left[\left(\sum_{j=1}^{n-1} e_{j} \partial_{j}-\partial_{n}\right) Z^{\beta-e^{i}}\right] z_{i}+\sum_{i=1}^{n-1}\left(\sum_{j=1}^{n-1} e_{j} Z^{\beta-e^{i}} \partial_{j} z_{i}-Z^{\beta-e^{i}} \partial_{n} z_{i}\right) \\
& =\sum_{i=1}^{n-1}\left(-\bar{\partial} Z^{\beta-e^{i}}\right) z_{i}+\sum_{i=1}^{n-1}\left(\sum_{j=1}^{n-1} e_{j} Z^{\beta-e^{i}} \delta_{i j}-Z^{\beta-e^{i}} e_{i}\right) \\
& =0+\sum_{i=1}^{n-1}\left(e_{i} Z^{\beta-e^{i}}-Z^{\beta-e^{i}} e_{i}\right) \quad \text { (by the induction hypothesis) } \\
& =\sum_{i \neq j} 2 e_{i} e_{j} \operatorname{comp}_{j} Z^{\beta-e^{i}} \\
& =\sum_{i<j} 2 e_{i} e_{j} \operatorname{comp}_{j} Z^{\beta-e^{i}}+\sum_{i>j} 2 e_{i} e_{j} \operatorname{comp}_{j} Z^{\beta-e^{i}} \\
& =\sum_{i<j} 2 e_{i} e_{j} \operatorname{comp}_{j} Z^{\beta-e^{i}}+\sum_{j>i} 2 e_{j} e_{i} \operatorname{comp}_{i} Z^{\beta-e^{j}} \\
& =\sum_{i<j} 2\left(e_{i} e_{j}+e_{j} e_{i}\right) \operatorname{comp}_{j} Z^{\beta-e^{i}} \quad(\text { by (3.3)) } \\
& =0 \quad(\text { by }(1.1)) \text {. }
\end{aligned}
$$

Hence $\bar{\partial} Z^{\beta}=0$.

Corollary 3.2. Components of symmetric powers $Z^{\beta}$ are homogeneous harmonic polynomials.

Proof. First, obviously, components of $Z^{\beta}$ are homogeneous polynomials by (3.1). Then, since $\bar{\partial} Z^{\beta}=0$, if we apply the Laplace operator $\Delta=\partial \bar{\partial}$ on $Z^{\beta}$, we have $\Delta\left(Z^{\beta}\right)=(\partial \bar{\partial}) Z^{\beta}=\partial\left(\bar{\partial} Z^{\beta}\right)=0$, and so all the components of $Z^{\beta}$ must be harmonic. 
Lemma 3.3. Symmetric powers satisfy the following differentiation formulas:

$$
\begin{gathered}
\partial_{i} Z^{\beta}=|\beta| Z^{\beta-e^{i}} \quad \text { for } 1 \leq i \leq n-1, \\
\partial_{n} Z^{\beta}=|\beta| \sum_{i=1}^{n-1} Z^{\beta-e^{i}} e_{i} .
\end{gathered}
$$

Proof. Formula (3.6) is proved by induction on $|\beta|$. For $|\beta|=1$, (3.6) holds quite trivially. So assume (3.6) with $|\beta|=m$ as the induction hypothesis and consider (3.6) with $|\beta|=m+1$. Now

$$
\begin{aligned}
\partial_{i} Z^{\beta} & =\partial_{i}\left(\sum_{j=1}^{n-1} z_{j} Z^{\beta-e^{j}}\right) \quad(\text { by }(3.1)) \\
& =\sum_{j=1}^{n-1}\left[\left(\partial_{i} z_{j}\right) Z^{\beta-e^{j}}+z_{j}\left(\partial_{i} Z^{\beta-e^{j}}\right)\right] \\
& =\sum_{j=1}^{n-1}\left(\delta_{i j} Z^{\beta-e^{j}}+z_{j}\left|\beta-e^{j}\right| Z^{\beta-e^{j}-e^{i}}\right) \quad \text { (by the induction hypothesis) } \\
& =Z^{\beta-e^{i}}+(|\beta|-1) \sum_{j=1}^{n-1} z_{j} Z^{\beta-e^{i}-e^{j}} \\
& =Z^{\beta-e^{i}}+(|\beta|-1) Z^{\beta-e^{i}} \quad \text { (again by (3.1)) } \\
& =|\beta| Z^{\beta-e^{i}},
\end{aligned}
$$

and (3.6) holds for $|\beta|=m+1$.

Formula (3.7) is proved by using (3.6) together with the fact that $Z^{\beta}$ being polycomplex and holomorphic is also retroholomorphic by Lemma 3.2. Indeed,

$$
\partial_{n} Z^{\beta}=Z^{\beta} \partial_{n}=Z^{\beta}\left(\bar{\partial}+\sum_{i=1}^{n-1} e_{i} \partial_{i}\right)=\sum_{i=1}^{n-1} \partial_{i} Z^{\beta} e_{i}=|\beta| \sum_{i=1}^{n-1} Z^{\beta-e^{i}} e_{i}
$$

As useful variations of (3.6) and (3.7) we have

$$
\begin{gathered}
\partial_{i}(Z-P)^{\beta}=|\beta|(Z-P)^{\beta-e^{i}} \quad \text { for } 1 \leq i \leq n-1, \\
\partial_{n}(Z-P)^{\beta}=|\beta| \sum_{i=1}^{n-1}(Z-P)^{\beta-e^{i}} e_{i},
\end{gathered}
$$

where the constant $P=\left(p_{1}, p_{2}, \ldots, p_{n-1}\right)=\left(a_{1}+e_{1} a_{n}, a_{2}+e_{2} a_{n}, \ldots, a_{n-1}+\right.$ $\left.e_{n-1} a_{n}\right)$ arises from a constant point $\mathbf{a}=\left(a_{1}, a_{2}, \ldots, a_{n}\right)$ in $R^{n}$, and similarly also

$$
\begin{gathered}
\partial_{i}(P-Z)^{\beta}=-|\beta|(P-Z)^{\beta-e^{i}} \text { for } 1 \leq i \leq n-1, \\
\partial_{n}(P-Z)^{\beta}=-|\beta| \sum_{i=1}^{n-1}(P-Z)^{\beta-e^{i}} e_{i} .
\end{gathered}
$$




\section{TAYLOR FORMULA}

A Taylor formula recovers a function from its partial derivatives of a certain order and values of all the lower order partial derivatives at a point, say the origin, combined with appropriate power functions. In the case of holomorphic functions in $R^{n}$, the required power functions are the symmetric powers $Z^{\beta}$.

In order to state our Taylor formula we shall need line integrals in $R^{n}$. Let

$$
f(\mathbf{x})=\sum_{\lambda} e_{\lambda} v_{\lambda}(\mathbf{x}) \quad \text { and } \quad g(\mathbf{x})=\sum_{\mu} e_{\mu} w_{\mu}(\mathbf{x})
$$

be smooth functions in some $\Omega$ in $R^{n}$, and let $C$ be a path of integration (having a continuously varying tangent vector) in $\Omega$, then we define two Stieltjes line inegrals of $f$ and $g$ along $C$ by

$$
\begin{aligned}
\int_{C} f(d g) & =\sum_{\lambda, \mu}\left(e_{\lambda} e_{\mu}\right) \int_{C} \sum_{i=1}^{n}\left[v_{\lambda}\left(\partial_{i} w_{\mu}\right)\right] d x_{i}, \\
\int_{C}(d f) g & =\sum_{\lambda, \mu}\left(e_{\lambda} e_{\mu}\right) \int_{C} \sum_{i=1}^{n}\left[\left(\partial_{i} v_{\lambda}\right) w_{\mu}\right] d x_{i} .
\end{aligned}
$$

Lemma 4.1 (integration-by-parts formulas). If $f$ and $g$ are smooth functions in some domain $\Omega$ in $R^{n}$, and $C$ is a path of integration in $\Omega$ going from a to $\mathbf{b}$, then

$$
\begin{aligned}
& \int_{C} f(d g)=[f(\mathbf{b}) g(\mathbf{b})-f(\mathbf{a}) g(\mathbf{a})]+\int_{C} d f(-g), \\
& \int_{C}(d f) g=\left[f(\mathbf{b})(g(\mathbf{b})-f(\mathbf{a}) g(\mathbf{a})]+\int_{C}(-f) d g .\right.
\end{aligned}
$$

Proof. With proper rearrangement of summations, (4.1) and (4.2) can be conveniently written as

$$
\begin{gathered}
\int_{C} f(d g)=\int_{C} f\left(\partial_{1} g\right) d x_{1}+f\left(\partial_{2} g\right) d x_{2}+\cdots+f\left(\partial_{n} g\right) d x_{n}, \\
\int_{C}(d f) g=\int_{C}\left(\partial_{1} f\right) g d x_{1}+\left(\partial_{2} f\right) g d x_{2}+\cdots+\left(\partial_{n} f\right) g d x_{n} .
\end{gathered}
$$

Adding these two equations, we have

$$
\int_{C} f(d g)+(d f) g=\int_{C} \partial_{1}(f g) d x_{1}+\cdots+\partial_{n}(f g) d x_{n} .
$$

But in view of the fundamental theorem of line integrals we also have

$$
\int_{C} \partial_{1}(f g) d x_{1}+\cdots+\partial_{n}(f g) d x_{n}=f(\mathbf{b}) g(\mathbf{b})-f(\mathbf{a}) g(\mathbf{a}),
$$

where $\mathbf{a}$ and $\mathbf{b}$ are the end points of the path of integration $C$. Combining (4.5) and (4.6), we have

$$
\int_{C} f(d g)+(d f) g=f(\mathbf{b}) g(\mathbf{b})-f(\mathbf{a}) g(\mathbf{a}) .
$$


Transposing the first and the second part of the integral, we obtain respectively (4.3) and (4.4).

Before we state our Taylor formula, we introduce the differential operator

$$
D^{\beta}=\left(\partial_{1}, \partial_{2}, \ldots, \partial_{n-1}\right)^{\left(\beta_{1}, \beta_{2}, \ldots, \beta_{n-1}\right)}=\partial_{1}^{\beta_{1}} \partial_{2}^{\beta_{2}} \cdots \partial_{n-1}^{\beta_{n-1}},
$$

in which $\partial_{n}$ is notably absent, and we shall keep in mind that the order of partial differentiations is immaterial for functions that are sufficiently smooth. And lastly, we will use the notation $\int_{\mathbf{a}}^{\mathbf{b}} f(\mathbf{x}) d g(\mathbf{x})$ whenever the integral is independent of particular choices of the path of integration going from $\mathbf{a}$ to $\mathbf{b}$, and also the notation $\int_{\mathbf{a}}^{\mathbf{x}} f(\tilde{\mathbf{x}}) d g(\tilde{\mathbf{x}})$ whenever $\mathbf{x}$ is preempted to denote an end point of the path of integration.

Theorem 4.1. If $f$ is holomorphic and sufficiently smooth in a domain $\Omega$ in $R^{n}$ containing the origin $\mathbf{0}$, then for any $\mathbf{x}$ in $\Omega$, we have

$$
\begin{aligned}
f(\mathbf{x})= & \sum_{|\beta|=0}^{m-1}\left[Z^{\beta} /|\beta| !\right] D^{\beta} f(\mathbf{0}) \\
& \left.+\sum_{|\beta|=m} \int_{0}^{\mathbf{x}} d\left[-(Z-\tilde{Z})^{\beta} / m !\right] D^{\beta} f(\tilde{\mathbf{x}}) \quad \text { (Taylor formula }\right),
\end{aligned}
$$

where $\tilde{Z}=Z(\tilde{\mathbf{x}})$ and the line integral is along any path of integration in $\Omega$ going from $\mathbf{0}$ to $\mathbf{x}$.

Proof. We prove the theorem by induction on $m$. First, if $m=1,(4.8)$ reduces to

$$
\begin{aligned}
f(\mathbf{x})-f(\mathbf{0}) & =\sum_{i=1}^{n-1} \int_{0}^{\mathbf{x}} d\left[-\left(z_{i}-\tilde{z}_{i}\right)\right] \partial_{i} f(\tilde{\mathbf{x}}) \\
& =\sum_{i=1}^{n-1} \int_{0}^{\mathbf{x}} d\left(\tilde{x}_{i}+e_{i} \tilde{x}_{n}\right) \partial_{i} f(\tilde{\mathbf{x}}) \quad\left(\text { since } z_{i} \text { remains constant }\right) \\
& =\sum_{i=1}^{n-1} \int_{0}^{\mathbf{x}} d \tilde{x}_{i} \partial_{i} f(\tilde{\mathbf{x}})+\int_{\mathbf{0}}^{\mathbf{x}} d \tilde{x}_{n}\left(\sum_{i=1}^{n-1} e_{i} \partial_{i}\right) f(\tilde{\mathbf{x}}) \\
& =\sum_{i=1}^{n} \int_{0}^{\mathbf{x}} d \tilde{x}_{i} \partial_{i} f(\tilde{\mathbf{x}}) \quad(\text { by holomorphy of } f),
\end{aligned}
$$

which is none other than the fundamental theorem of line integrals in $R^{n}$.

Next we take (4.8) as our induction hypothesis and show that (4.8) remains valid when $m$ is replaced throughout by $m+1$. For this we focus on the remainder term in (4.8) and apply the integration-by-parts formula (4.4), keeping in mind that if the line integral on one side of the formula is independent of the paths of integration then so is the line integral on the other side. The remainder term then splits into two terms:

$$
\begin{aligned}
& \sum_{|\beta|=m} \int_{\mathbf{0}}^{\mathbf{x}} d\left[-(Z-\tilde{Z})^{\beta} / m !\right] D^{\beta} f(\tilde{\mathbf{x}}) \\
& \quad=\sum_{|\boldsymbol{\beta}|=m}\left[Z^{\beta} / m !\right] D^{\beta} f(\mathbf{0})+\sum_{|\beta|=m} \int_{0}^{\mathbf{x}}\left[(Z-\tilde{Z})^{\beta} / m !\right] d\left[D^{\beta} f(\tilde{\mathbf{x}})\right] .
\end{aligned}
$$


Now, while we let the first term join the cumulative summation in (4.8), we work on the last part of the second term:

$$
\begin{aligned}
d\left[D^{\beta} f(\tilde{\mathbf{x}})\right] & \left.=\sum_{i=1}^{n-1} D^{\beta+e^{i}} f(\tilde{\mathbf{x}}) d \tilde{x}_{i}+D^{\beta}\left(\sum_{i=1}^{n-1} e_{i} \partial_{i}\right) f(\tilde{\mathbf{x}}) d \tilde{x}_{n} \quad \text { (since } \bar{\partial} f=0\right) \\
& =\sum_{i=1}^{n-1}\left(d \tilde{x}_{i}+e_{i} d \tilde{x}_{n}\right) D^{\beta+e^{i}} f(\tilde{\mathbf{x}})=\sum_{i=1}^{n-1} d \tilde{z}_{i} D^{\beta+e^{i}} f(\tilde{\mathbf{x}}) .
\end{aligned}
$$

Consequently, we can rewrite the second term as:

$$
\begin{aligned}
& \sum_{|\beta|=m} \int_{0}^{\mathbf{x}}\left[(Z-\tilde{Z})^{\beta} / m !\right]\left[\sum_{i=1}^{n-1} d \tilde{z}_{i} D^{\beta+e^{i}} f(\tilde{\mathbf{x}})\right] \\
& =\sum_{|\beta|=m} \sum_{i=1}^{n-1} \int_{0}^{\mathbf{x}}\left[(Z-\tilde{Z})^{\beta} / m !\right] d \tilde{z}_{i}\left[D^{\beta+e^{i}} f(\tilde{\mathbf{x}})\right] \\
& =\sum_{|\gamma|=m+1} \int_{0}^{\mathbf{x}} \sum_{i=1}^{n-1}\left[(Z-\tilde{Z})^{\gamma-e^{i}} / m !\right] d \tilde{z}_{i}\left[D^{\gamma} f(\tilde{\mathbf{x}})\right] .
\end{aligned}
$$

Finally, leaving the last part of the last integral intact, we attempt to show

$$
\sum_{i=1}^{n-1}\left[(Z-\tilde{Z})^{\gamma-e^{i}} / m !\right] d \tilde{z}_{i}=d\left[-(Z-\tilde{Z})^{\gamma} /(m+1) !\right]
$$

in order to arrive at the correct remainder term. In fact, we choose to show this last equation backwards by using differentiation formulas (3.10) and (3.11). Indeed, with $|\gamma|=m+1$ we have

$$
\begin{aligned}
d[- & \left.(Z-\tilde{Z})^{\gamma} /(m+1) !\right] \\
& =\sum_{i=1}^{n-1}\left[(Z-\tilde{Z})^{\gamma-e^{i}} / m !\right] d \tilde{x}_{i}+\sum_{i=1}^{n-1}\left[(Z-\tilde{Z})^{\gamma-e^{i}} / m !\right] e_{i} d \tilde{x}_{n} \\
& =\sum_{i=1}^{n-1}\left[(Z-\tilde{Z})^{\gamma-e^{i}} / m !\right] d\left(\tilde{x}_{i}+e_{i} \tilde{x}_{n}\right) \\
& =\sum_{i=1}^{n-1}\left[(Z-\tilde{Z})^{\gamma-e^{i}} / m !\right] d \tilde{z}_{i} .
\end{aligned}
$$

Formula (4.8) can be modified by a slight change in notation. For instance, although $\mathbf{x}=\left(x_{1}, x_{2}, \ldots, x_{n}\right)$ is the ultimate independent variable in $R^{n}$, it can be uniquely represented by $Z=\left(z_{1}, z_{2}, \ldots, z_{n-1}\right)$. Formula (4.8) then becomes

$$
\begin{aligned}
f(Z)= & \sum_{|\beta|=0}^{m-1}\left[Z^{\beta} /|\beta| !\right] D^{\beta} f(0) \\
& +\sum_{|\beta|=m} \int_{0}^{Z} d\left[-(Z-\widetilde{Z})^{\beta} / m !\right] D^{\beta} f(\tilde{Z}) \quad\left(\text { Taylor formula in } R^{n}\right) .
\end{aligned}
$$


In particular, if $n=2, Z$ reduces to $z_{1}=x_{1}+e_{1} x_{2}=x+i y=z, \beta$ reduces to $\beta_{1}=k$, and $D$ reduces to $\partial_{1}=\partial / \partial x=d / d z$ for holomorphic functions in $R^{2}$, and so (4.9) reduces to

$$
\begin{aligned}
f(z)= & \sum_{k=0}^{m-1}\left(z^{k} / k !\right) f^{(k)}(0) \\
& +\int_{0}^{z} d\left[-(z-\tilde{z})^{m} / m !\right] f^{(m)}(\tilde{x}) \quad\left(\text { Taylor formula in } R^{2}\right) .
\end{aligned}
$$

Further, since

$$
\begin{aligned}
d\left[-(z-\tilde{z})^{m} / m !\right] & =\partial_{1}\left[-(z-\tilde{z})^{m} / m !\right] d \tilde{x}+\partial_{2}\left[-(z-\tilde{z})^{m} / m !\right] d \tilde{y} \\
& =\left[(z-\tilde{z})^{m-1} /(m-1) !\right] d \tilde{x}+\left[(z-\tilde{z})^{m-1} /(m-1) !\right] i d \tilde{y} \\
& =\left[(z-\tilde{z})^{m-1} /(m-1) !\right] d \tilde{z},
\end{aligned}
$$

hence, (4.10) becomes, after replacing $m$ throughout by $m+1$,

$$
f(z)=\sum_{k=0}^{m}\left[f^{(k)}(0) / k !\right] z^{k}+\int_{0}^{z}\left[f^{(m+1)}(\tilde{z}) / m !\right](z-\tilde{z})^{m} d \tilde{z},
$$

which resembles the familiar Taylor formula found in elementary calculus.

\section{CAUCHY INTEgRAL FORMULA}

We consider only domains that are regular, that is, open connected sets in $R^{n}$ on which the divergence theorem holds. A hyperball, for example, is such a domain. Specifically, if $\Omega$ is regular, then for any real function $u(\mathbf{x})$ continuously differentiable in $\Omega$ and continuous on the closure $\bar{\Omega}$, we have

$$
\iint_{\partial \boldsymbol{\Omega}} \nu_{i}(\boldsymbol{\xi}) u(\boldsymbol{\xi}) d \boldsymbol{\xi}=\iiint_{\Omega} \partial_{i} u(\mathbf{x}) d \mathbf{x} \text { for } 1 \leq i \leq n,
$$

where $\left(\nu_{1}, \nu_{2}, \ldots, \nu_{n}\right)=\boldsymbol{\nu}$ is the unit normal vector on the hypersurface $\partial \Omega$ pointing outward, $\boldsymbol{\xi}$ is the variable representing points on the boundary $\partial \Omega$, and $\mathbf{x}$ is the variable representing points in $\Omega$. Formula (5.1) is a useful reduced form of the well-known Gauss divergence theorem, which can be restored from (5.1) by replacing $u$ with $u_{i}$ and summing over $1 \leq i \leq n$. Now an extensive linear recombination of (5.1) after replacing $u$ with $v_{\lambda}$ leads to a quasidivergence theorem (5.3) below, out of which emerges a Cauchy integral theorem.

Theorem 5.1 (Cauchy integral theorem). Let $f(\mathbf{x})=\sum_{\lambda} e_{\lambda} v_{\lambda}(\mathbf{x})$ be continuously differentiable with $\bar{\partial} f=0$ in a regular domain $\Omega$ in $R^{n}$ and continuous on the closure $\bar{\Omega}$, then

$$
\iint_{\partial \Omega} \bar{\nu}(\boldsymbol{\xi}) f(\boldsymbol{\xi}) d \boldsymbol{\xi}=0 \quad \text { (Cauchy integral theorem in } R^{n} \text { ) }
$$

where $\bar{\nu}$ is the conjugate of the polycomplex unit normal $\nu=e_{1} \nu_{1}+e_{2} \nu_{2}+\cdots+$ $e_{n-1} \nu_{n-1}+\nu_{n}$ formed by combining the components of the outward unit normal vector $\nu=\left(\nu_{1}, \nu_{2}, \ldots, \nu_{n}\right)$.

Proof. Taking a component from $\nu$ and a component from $f$, we have in view of $(5.1)$

$$
\iint_{\partial \Omega} \nu_{i} v_{\lambda} d \boldsymbol{\xi}=\iiint_{\Omega} \partial_{i} v_{\lambda} d \mathbf{x}
$$


Multiplying both sides by $\bar{e}_{i} e_{\lambda}$ and summing over $i$ and $\lambda$, we obtain

$$
\sum_{i=1}^{n} \sum_{\lambda}\left(\bar{e}_{i} e_{\lambda}\right) \iint_{\partial \Omega} \nu_{i} v_{\lambda} d \xi=\sum_{i=1}^{n} \sum_{\lambda}\left(\bar{e}_{i} e_{\lambda}\right) \iiint_{\Omega} \partial_{i} v_{\lambda} d \mathbf{x}
$$

Hence, by linearity of integrals, we have

$$
\iint_{\partial \Omega} \bar{\nu} f d \boldsymbol{\xi}=\iiint_{\Omega} \bar{\partial} f d \mathbf{x}
$$

Now substituting $\bar{\partial} f=0$ in the last integral, we obtain (5.2).

In particular, if $n=2$, then the surface integral reduces to the line integral $\oint_{\partial \Omega} \bar{\nu} f d s$, but $\bar{\nu} d s=\left(-i \nu_{1}+\nu_{2}\right) d s=-\left(-\nu_{2}+i \nu_{1}\right) d s=-(d x+i d y)=-d z$ since $\left(-\nu_{2}, \nu_{1}\right)$ is the unit tangent vector obtained by a 90 degree counterclockwise rotation of the outward unit normal vector $\left(\nu_{1}, \nu_{2}\right)$, therefore (5.2) reduces to

$$
\oint_{\partial \Omega} f d z=0 \quad \text { (Cauchy integral theorem in } R^{2} \text { ). }
$$

A generalization of (5.2) is now necessary in order to deduce from it a Cauchy integral formula. We follow examples of Delanghe [2] and Hile [5] and augment the integrand $\bar{\nu} f$ in (5.2) to $g \bar{\nu} f$.

Theorem 5.2 (generalized Cauchy integral theorem). If $f(\mathbf{x})=\sum_{\lambda} e_{\lambda} v_{\lambda}(\mathbf{x})$ and $g(\mathbf{x})=\sum_{\mu} e_{\mu} w_{\mu}(\mathbf{x})$ are continuously differentiable with $\bar{\partial} f=0$ and $g \bar{\partial}=0$ in a regular domain $\Omega$ in $R^{n}$ and are continuous on the closure $\bar{\Omega}$, then

$$
\iint_{\partial \Omega} g(\boldsymbol{\xi}) \bar{\nu}(\boldsymbol{\xi}) f(\boldsymbol{\xi}) d \boldsymbol{\xi}=0 .
$$

Proof. First without assuming $\bar{\partial} f=g \bar{\partial}=0$, we work out in general

$$
\begin{aligned}
\iint_{\partial \Omega} g \nu f d \boldsymbol{\xi} & =\iint_{\partial \Omega}\left(\sum_{\mu} e_{\mu} w_{\mu}\right)\left(\sum_{i=1}^{n} e_{i} \nu_{i}\right)\left(\sum_{\lambda} e_{\lambda} v_{\lambda}\right) d \xi \\
& =\sum_{\lambda, \mu} \sum_{i=1}^{n}\left(e_{\mu} e_{i} e_{\lambda}\right) \iint_{\partial \Omega} \nu_{i} w_{\mu} v_{\lambda} d \xi .
\end{aligned}
$$

But by (5.1) we have

$$
\iint_{\partial \Omega} \nu_{i}\left(w_{\mu} v_{\lambda}\right) d \boldsymbol{\xi}=\iiint_{\Omega} \partial_{i}\left(w_{\mu} v_{\lambda}\right) d \mathbf{x}=\iiint_{\Omega}\left[\left(\partial_{i} w_{\mu}\right) v_{\lambda}+w_{\mu}\left(\partial_{i} v_{\lambda}\right)\right] d \mathbf{x}
$$

Therefore we obtain

$$
\begin{aligned}
\iint_{\partial \Omega} g \nu f d \boldsymbol{\xi} & =\iiint_{\Omega}\left[\sum_{i} \partial_{i}\left(\sum_{\mu} e_{\mu} w_{\mu}\right) e_{i}\right]\left(\sum_{\lambda} e_{\lambda} v_{\lambda}\right) \\
& +\left(\sum_{\mu} e_{\mu} w_{\mu}\right)\left[\left(\sum_{i} e_{i} \partial_{i}\right)\left(\sum_{\lambda} e_{\lambda} v_{\lambda}\right)\right] d \mathbf{x} \\
= & \iiint_{\Omega}[(g \partial) f+g(\partial f)] d \mathbf{x} .
\end{aligned}
$$


Replacing $\nu$ by $\bar{\nu}$, we can likewise show

$$
\iint_{\partial \Omega} g \bar{\nu} f d \boldsymbol{\xi}=\iiint_{\Omega}[(g \bar{\partial}) f+g(\bar{\partial} f)] d \mathbf{x} .
$$

Finally, substituting $\bar{\partial} f=g \bar{\partial}=0$ in the last integral, we obtain (5.5).

Corollary 5.2. If $f$ and $g$ are polycomplex and continuously differentiable and holomorphic in a regular domain $\Omega$ in $R^{n}$ and are continuous on the closure $\bar{\Omega}$, then

$$
\iint_{\partial \Omega} g(\boldsymbol{\xi}) \bar{\nu}(\boldsymbol{\xi}) f(\boldsymbol{\xi}) d \boldsymbol{\xi}=0
$$

Proof. $g$ being polycomplex and holomorphic is in fact retroholomorphic by Theorem 2.2. Therefore conditions in Theorem 5.2 are satisfied, and (5.7) follows.

We deduce a Cauchy integral formula from (5.5) by first transforming it into (5.8) below.

Theorem 5.3 (principle of deformation). Let $S_{1}$ enclosing $S_{2}$ be two closed surfaces that together form the boundary of some regular domain $\Omega^{*}$, inside which $f$ and $g$ are continuously differentiable with $\bar{\partial} f=g \bar{\partial}=0$, and over the closure of which $f$ and $g$ are continuous, then

$$
\iint_{S_{1}} g(\boldsymbol{\xi}) \bar{\nu}(\boldsymbol{\xi}) f(\boldsymbol{\xi}) d \boldsymbol{\xi}=\iint_{S_{2}} g(\boldsymbol{\xi}) \bar{\nu}(\boldsymbol{\xi}) f(\boldsymbol{\xi}) d \boldsymbol{\xi},
$$

where $\bar{\nu}$ denotes the conjugate of the polycomplex outward unit normal $\nu$ of the closed surface in each of the two surface integrals.

Proof. Since any outward normal of $S_{2}$ is an inward normal of $\partial \Omega^{*}$, we have

$$
\iint_{\partial \Omega^{*}} g \bar{\nu} f d \xi=\iint_{S_{1}} g \bar{\nu} f d \xi-\iint_{S_{2}} g \bar{\nu} f d \xi
$$

But the integral on the left is zero by (5.5), so (5.8) is valid.

Equation (5.8) leads to Cauchy integral formula (5.9) below if, while taking $S_{1}=\partial \Omega$ and $f$ holomorphic in $\Omega$ as given, we let $g$ be a suitable function to play the role of Cauchy kernel and $S_{2}$ be a small sphere to facilitate the evaluation of the surface integral.

Theorem 5.4. Let $f(\mathbf{x})=\sum_{\lambda} e_{\lambda} v_{\lambda}(\mathbf{x})$ be continuously differentiable and holomorphic in a regular domain $\Omega$ in $R^{n}$ and continuous over the closure $\bar{\Omega}$, then for any $\mathbf{x}$ in $\Omega$, we have

$$
f(\mathbf{x})=\omega_{n}^{-1} \iint_{\partial \Omega}\|\boldsymbol{\xi}-\mathbf{x}\|^{-n}(\xi-x) \bar{\nu}(\boldsymbol{\xi}) f(\boldsymbol{\xi}) d \boldsymbol{\xi}
$$

(Cauchy integral formula),

where $x=e_{1} x_{1}+\cdots+e_{n-1} x_{n-1}+x_{n}, \xi=e_{1} \xi_{1}+\cdots+e_{n-1} \xi_{n-1}+\xi_{n}, \omega_{n}$ is the area of the unit sphere in $R^{n}$, and $\bar{\nu}(\xi)$ is the conjugate of the polycomplex outward unit normal $\nu$ of $\partial \Omega$ at $\boldsymbol{\xi}$.

Proof. Let $g$ be the polycomplex function defined by

$$
g(\boldsymbol{\xi}, \mathbf{x})=\|\boldsymbol{\xi}-\mathbf{x}\|^{-n}(\boldsymbol{\xi}-x) \quad \text { (Cauchy kernel) }
$$


with $\mathbf{x}=\left(x_{1}, x_{2}, \ldots, x_{n}\right)$ and $\boldsymbol{\xi}=\left(\xi_{1}, \xi_{2}, \ldots, \xi_{n}\right)$ both in $\bar{\Omega}$, and

$$
\begin{aligned}
& x=x(\mathbf{x})=e_{1} x_{1}+e_{2} x_{2}+\cdots+e_{n-1} x_{n-1}+x_{n}, \\
& \xi=\xi(\xi)=e_{1} \xi_{1}+e_{2} \xi_{2}+\cdots+e_{n-1} \xi_{n-1}+\xi_{n},
\end{aligned}
$$

then, for each fixed $\mathbf{x}, g$ can be shown by routine calculations to be holomorphic (and hence also retroholomorphic by Theorem 2.2) in $\boldsymbol{\xi} \in \Omega-\{\mathbf{x}\}$, and likewise, for each fixed $\xi, g$ can be shown to be holomorphic (and hence again retroholomorphic) in $\mathbf{x} \in \Omega-\{\boldsymbol{\xi}\}$.

Substituting (5.10) in (5.8) and letting $S_{1}=\partial \Omega$, while choosing $S_{2}$ to be the boundary of a small ball $B_{\rho}(\mathbf{x})$ of radius $\rho>0$ centered at the point $\mathbf{x} \in \Omega$, we obtain

$$
\iint_{\partial \Omega} g(\boldsymbol{\xi}, \mathbf{x}) \bar{\nu}(\boldsymbol{\xi}) f(\boldsymbol{\xi}) d \boldsymbol{\xi}=\iint_{\partial B_{\rho}(\mathbf{x})} g(\boldsymbol{\xi}, \mathbf{x}) \bar{\nu}(\boldsymbol{\xi}) f(\boldsymbol{\xi}) d \boldsymbol{\xi} .
$$

Now noting that on $\partial B_{\rho}(\mathbf{x}) \bar{\nu}(\boldsymbol{\xi})=\|\boldsymbol{\xi}-\mathbf{x}\|^{-1}(\bar{\xi}-\bar{x})$ and $|\xi-x|=\rho$, we see the surface integral on $\partial B_{\rho}(\mathbf{x})$ can be simplified after some routine calculations to

$$
\iint_{\partial B_{\rho}(\mathbf{x})} g(\boldsymbol{\xi}, \mathbf{x}) \bar{\nu}(\boldsymbol{\xi}) f(\boldsymbol{\xi}) d \boldsymbol{\xi}=\rho^{-n+1} \iint_{\partial B_{\rho}(\mathbf{x})} f(\boldsymbol{\xi}) d \boldsymbol{\xi} .
$$

But if $\omega_{n}$ denotes the area of a unit sphere in $R^{n}$, then the area of $\partial B_{\rho}(\mathbf{x})$ is given by $\omega_{n} \rho^{n-1}$, and the last surface integral above reduces to $\omega_{n} \rho^{n-1}$ multiplied by the average value of $f$ on $\partial B_{\rho}(\mathbf{x})$, which tends to $f(\mathbf{x})$ by continuity of $f$ at $\mathbf{x}$ as $\rho$ approaches 0 . Consequently, (5.11) and (5.12) give rise to (5.9).

Formula (5.9) can be modified by a slight change in notation. If we let $x$ and $\xi$ represent $\mathbf{x}$ and $\boldsymbol{\xi}$, then (5.9) becomes

$$
f(x)=\omega_{n}^{-1} \iint_{\partial \Omega}|\xi-x|^{-n}(\xi-x) \bar{\nu}(\xi) f(\xi) d \xi
$$

(Cauchy integral formula in $R^{n}$ ),

where the Cauchy kernel is now expressed as

$$
g(\xi, x)=|\xi-x|^{-n}(\xi-x) .
$$

In particular, if $n=2$, then $\omega_{2}=2 \pi$, and " $x$ " reduces to $e_{1} x_{1}+x_{2}=i x+y=$ $i(x-i y)=i \bar{z}$, and likewise, " $\xi$ " reduces to $i \bar{\zeta}$. Also $\bar{\nu} d \xi$ reduces to $-d \zeta$ as was shown in the derivation of (5.4). Consequently (5.13) reduces to

$$
\begin{aligned}
f(i \bar{z}) & =(2 \pi)^{-1} \int_{\partial \Omega}|i \bar{\zeta}-i \bar{z}|^{-2}(i \bar{\zeta}-i \bar{z}) f(i \bar{\zeta})(-d \zeta) \\
& =(2 \pi i)^{-1} \int_{\partial \Omega}(\zeta-z)^{-1} f(i \bar{\zeta}) d \zeta
\end{aligned}
$$

Now the ultimate independent variable $(x, y)$ in $R^{2}$ can be represented by $i \bar{z}$ or $z$ as long as $f(i \bar{z})$ and $f(z)$ are understood to represent the same mapping of $\Omega$. We may therefore rewrite the above equation as

(5.15) $f(z)=(2 \pi i)^{-1} \int_{\partial \Omega}(\zeta-z)^{-1} f(\zeta) d \zeta \quad$ (Cauchy integral formula in $R^{2}$ ).

\section{TAYLOR SERIES EXPANSIONS}

We now apply our Taylor formula (4.8) to Cauchy kernel (5.14) and analyze the remainder term to arrive at Taylor expansions of holomorphic functions. 
Theorem 6.1. If $f(\mathbf{x})=\sum_{\lambda} e_{\lambda} v_{\lambda}(\mathbf{x})$ is continuously differentiable and holomorphic in a spherical domain $\|\mathbf{x}\|<a$ in $R^{n}$ and continuous on its closure $\|\mathbf{x}\| \leq a$, then $f(\mathbf{x})$ can be expanded in power series

$$
f(\mathbf{x})=\sum_{|\beta|=0}^{\infty}\left[Z^{\beta} /|\beta| !\right] D^{\beta} f(\mathbf{0}),
$$

which converges uniformly for $\|\mathbf{x}\| \leq a / 4$.

Proof. Whenever convenient we shall write $x$ and $\xi$ for $\mathbf{x}$ and $\xi$. First by applying the Taylor formula (4.8) to the Cauchy kernel $g(\xi, x)$ in (5.14) with $\xi$ held constant, we have

$$
g(\xi, x)=\left.\sum_{|\beta|=0}^{m-1}\left[Z^{\beta} /|\beta| !\right] D^{\beta} g(\xi, x)\right|_{x=0}+R_{m}(\xi, x),
$$

where

$$
R_{m}(\xi, x)=\sum_{|\beta|=m} \int_{0}^{x} d\left[-(Z-\tilde{Z})^{\beta} / m !\right] \widetilde{D}^{\beta} g(\xi, \tilde{x}) .
$$

Substituting (6.2) and (6.3) for the Cauchy kernel in (5.13) while taking $\Omega=B$, the spherical domain $\|\mathbf{x}\|<a$, we obtain

$$
f(x)=\left.\sum_{|\beta|=0}^{m-1}\left[Z^{\beta} /|\beta| !\right]\left[\omega_{n}^{-1} \iint_{\partial B} D^{\beta} g(\xi, x) \bar{\nu}(\xi) f(\xi) d \xi\right]\right|_{x=0}+\widehat{R}_{m}(x)
$$

where

$$
\widehat{R}_{m}(x)=\omega_{n}^{-1} \iint_{\partial B} R_{m}(\xi, x) \bar{\nu}(\xi) f(\xi) d \xi .
$$

But $D^{\beta}$ in (6.4) may be moved forward across the integral sign by Leibniz's rule justified by continuities of $D^{\beta} g$. With simplification by the Cauchy integral formula (5.13), we have

$$
f(x)=\sum_{|\beta|=0}^{m-1}\left[Z^{\beta} /|\beta| !\right] D^{\beta} f(0)+\widehat{R}_{m}(x) .
$$

Finally, since by Lemma 7.5 to follow $\left|\widehat{R}_{m}(x)\right|$ tends to zero uniformly for $\|\mathbf{x}\|<a / 4$ as $m$ goes to infinity, we obtain (6.1) with uniform convergence for $\|\mathbf{x}\|<a / 4$, and hence, by continuity of $f$, on the closure $\|\mathbf{x}\| \leq a / 4$.

In the Taylor series expansion (6.1) if we use $Z$ to represent $\mathbf{x}$, then (6.1) becomes

$$
f(Z)=\sum_{|\beta|=0}^{\infty}\left(Z^{\beta} /|\beta| !\right) D^{\beta} f(0) \quad\left(\text { Taylor expansion in } R^{n}\right) .
$$

In particular, if $n=2$, then as before, $Z$ reduces to $z_{1}=x_{1}+e_{1} x_{2}=x+i y=$ $z$, and $D^{\beta}$ reduces to $\partial^{k} / \partial x^{k}$ where $k=|\beta|$, and since $\partial / \partial x$ is equivalent to $d / d z$ for holomorphic functions in $R^{2}$, we obtain

$$
\left.f(z)=\sum_{k=0}^{\infty}\left(z^{k} / k !\right) f^{(k)}(0) \quad \text { (Taylor expansion in } R^{2}\right) .
$$




\section{REMAINDER TERMS}

Since the remainder term $\widehat{R}_{m}(x)$ arises in (6.5) from the remainder term $R_{m}(\xi, x)$, we begin by estimating the latter.

Lemma 7.1. If $R_{m}(\xi, x)$ is as given in (6.3), then

$$
R_{m}(\xi, x)=\int_{0}^{1}\left[(1-\tilde{t})^{m-1} /(m-1) !\right](\mathbf{x} \cdot \tilde{\boldsymbol{\partial}})^{m} g(\xi, \tilde{x}) d \tilde{t},
$$

where $\mathbf{x} \cdot \tilde{\boldsymbol{\partial}}=x_{1} \tilde{\partial}_{1}+x_{2} \tilde{\partial}_{2}+\cdots+x_{n} \tilde{\partial}_{n}$, and $\tilde{x}=x \tilde{t}$ for $0 \leq \tilde{t} \leq 1$.

Proof. For the line integral in (6.3) we choose as the path of integration the line segment from $\mathbf{0}$ to $\mathbf{x}$ and parameterize it by $\tilde{\mathbf{x}}=\mathbf{x} \tilde{t}$ with $0 \leq \tilde{t} \leq 1$, then

$$
(Z-\tilde{Z})^{\beta}=(Z-Z \tilde{t})^{\beta}=[(1-\tilde{t}) Z]^{\beta}=(1-\tilde{t})^{|\beta|} Z^{\beta},
$$

and therefore

$$
R_{m}(\xi, x)=\int_{0}^{1} d\left[-(1-\tilde{t})^{m} / m !\right]\left(\sum_{|\beta|=m} Z^{\beta} \widetilde{D}^{\beta}\right) g(\xi, \tilde{x}) .
$$

Now the operator on $g$ can be simplified in two steps to $(\mathbf{x} \cdot \tilde{\boldsymbol{\partial}})^{m}$. First we have

$$
\sum_{|\beta|=m} Z^{\beta} \widetilde{D}^{\beta}=\left(z_{1} \tilde{\partial}_{1}+\cdots+z_{n-1} \tilde{\partial}_{n-1}\right)^{m}
$$

which can be shown by induction on $m$. Indeed for $m=1$, we have trivially

$$
\sum_{|\beta|=1} Z^{\beta} \widetilde{D}^{\beta}=z_{1} \tilde{\partial}_{1}+\cdots+z_{n-1} \tilde{\partial}_{n-1}
$$

so assume (7.3) valid and proceed to

$$
\begin{aligned}
\sum_{|\beta|=m+1} Z^{\beta} \widetilde{D}^{\beta} & =\sum_{|\beta|=m+1}\left(z_{1} Z^{\beta-e^{1}}+\cdots+z_{n-1} Z^{\beta-e^{n-1}}\right) \widetilde{D}^{\beta} \quad(\text { by }(3.1)) \\
& =\sum_{|\beta|=m+1}\left(z_{1} \tilde{\partial}_{1} Z^{\beta-e^{1}} \widetilde{D}^{\beta-e^{1}}+\cdots+z_{n-1} \tilde{\partial}_{n-1} Z^{\beta-e^{n-1}} \widetilde{D}^{\beta-e^{n-1}}\right) .
\end{aligned}
$$

But for each $i$ we have

$$
\sum_{|\beta|=m+1} Z^{\beta-e^{i}} \widetilde{D}^{\beta-e^{i}}=\sum_{|\beta|=m} Z^{\beta} \widetilde{D}^{\beta}
$$


which by the induction hypothesis is equal to $\left(z_{1} \tilde{\partial}_{1}+\cdots+z_{n-1} \tilde{\partial}_{n-1}\right)^{m}$. Consequently,

$$
\sum_{|\beta|=m+1} Z^{\beta} \tilde{D}^{\beta}=\left(z_{1} \tilde{\partial}_{1}+\cdots+z_{n-1} \tilde{\partial}_{n-1}\right)^{m+1},
$$

and (7.3) is confirmed for all values of $m \geq 1$.

Next we see that

$$
\begin{aligned}
z_{1} \tilde{\partial}_{1}+ & \cdots+z_{n-1} \tilde{\partial}_{n-1}=\left(x_{1}+e_{1} x_{n}\right) \tilde{\partial}_{1}+\cdots+\left(x_{n-1}+e_{n-1} x_{n}\right) \tilde{\partial}_{n-1} \\
& =x_{1} \tilde{\partial}_{1}+\cdots+x_{n-1} \tilde{\partial}_{n-1}+x_{n}\left(e_{1} \tilde{\partial}_{1}+\cdots+e_{n-1} \tilde{\partial}_{n-1}\right) \\
& =x_{1} \tilde{\partial}_{1}+\cdots+x_{n-1} \tilde{\partial}_{n-1}+x_{n} \tilde{\partial}_{n} \\
& =\mathbf{x} \cdot \tilde{\boldsymbol{\partial}}
\end{aligned}
$$

so long as the operator is applied on holomorphic functions, for then $e_{1} \tilde{\partial}_{1}$ $+\cdots+e_{n-1} \tilde{\partial}_{n-1}=\tilde{\partial}_{n}$. Substituting (7.4) into (7.3) and then into (7.2), we obtain (7.1).

Lemma 7.2. If $g(\xi, x)=|\xi-x|^{-n}(\xi-x)$, then for $m \geq 1$

$$
\begin{aligned}
(\mathbf{x} \cdot \tilde{\boldsymbol{\partial}})^{m} g(\xi, \tilde{x})= & \sum_{j=0}^{[m / 2]} b_{m-2 j}^{m} \widetilde{A}^{-n-2 m+2 j} \widetilde{B}^{m-2 j}\left(-C^{2}\right)^{j}(\xi-\tilde{x}) \\
& +\sum_{j=0}^{(m / 2)} b_{m-2 j-1}^{m} \widetilde{A}^{-n-2 m+2 j+2} \widetilde{B}^{m-2 j-1}\left(-C^{2}\right)^{j} x,
\end{aligned}
$$

where $[m / 2]$ and $(m / 2)$ denote respectively the largest integer no greater than and the largest integer strictly less than $m / 2$, and $\widetilde{A}, \widetilde{B}, C$ are scalars given by $|\xi-\tilde{x}|, \mathbf{x} \cdot(\boldsymbol{\xi}-\tilde{\mathbf{x}}),|x|$, and $b_{i}^{m}$ are positive real coefficients satisfying the common inequality

$$
b_{i}^{m} \leq \prod_{k=1}^{m}(n+3 k) \quad \text { for } 0 \leq i \leq m .
$$

Proof. Since $g(\xi, \tilde{x})=|\xi-\tilde{x}|^{-n}(\xi-\tilde{x})=\tilde{A}^{-n}(\xi-\tilde{x})$, calculations of $(\mathbf{x} \cdot \widetilde{\boldsymbol{\partial}})^{m} g(\xi, \tilde{x})$ will be based on the following formulas that can be routinely checked:

$$
\begin{array}{ll}
(\mathbf{x} \cdot \tilde{\boldsymbol{\partial}}) \tilde{A}^{-k}=k \tilde{A}^{-k-2} \widetilde{B}, & \text { where } \widetilde{B}=\mathbf{x} \cdot(\boldsymbol{\xi}-\tilde{\mathbf{x}}), \\
(\mathbf{x} \cdot \tilde{\boldsymbol{\partial}}) \widetilde{B}^{k}=k \widetilde{B}^{k-1}\left(-C^{2}\right), & \text { where } C=\|\mathbf{x}\|=|x|, \\
(\mathbf{x} \cdot \tilde{\boldsymbol{\partial}})(\xi-\tilde{\boldsymbol{x}})=x . &
\end{array}
$$

Now for $m=1,(7.5)$ reduces to

$$
(\mathbf{x} \cdot \tilde{\boldsymbol{\partial}}) g(\xi, \tilde{x})=b_{1}^{1} \tilde{A}^{-n-2} \widetilde{B}(\xi-\tilde{x})+b_{0}^{1} \tilde{A}^{-n} x,
$$

which can be confirmed by using (7.7) and (7.9), for indeed

$$
(\mathbf{x} \cdot \tilde{\boldsymbol{\partial}}) g(\xi, \tilde{x})=(\mathbf{x} \cdot \tilde{\boldsymbol{\partial}}) \tilde{A}^{-n}(\xi-\tilde{x})=n \tilde{A}^{-n-2} \widetilde{B}(\xi-\tilde{x})+\tilde{A}^{-n} x .
$$

So (7.5) is valid for $m=1$ with $b_{1}^{1}=n$ and $b_{0}^{1}=1$, and (7.6) is also valid for $m=1$ since 


$$
b_{1}^{1}=n \leq n+3=\prod_{k=1}^{1}(n+3 k),
$$

and

$$
b_{0}^{1}=1 \leq n+3=\prod_{k=1}^{1}(n+3 k) \text {. }
$$

Therefore, we assume (7.5) and (7.6) as induction hypotheses and proceed to $(\mathbf{x} \cdot \tilde{\boldsymbol{\partial}})^{m+1} g(\xi, \tilde{x})$ and $b_{i}^{m+1}$ for $0 \leq i \leq m+1$. If we apply $\mathbf{x} \cdot \tilde{\boldsymbol{\partial}}$ to both sides of (7.5), we obtain

$$
\begin{aligned}
(\mathbf{x} \cdot \tilde{\boldsymbol{\partial}})^{m+1} g(\xi, \tilde{x}) & \\
= & \sum_{j=0}^{[m / 2]} b_{m-2 j}^{m}(n+2 m-2 j) \tilde{A}^{-n-2 m+2 j-2} \widetilde{B}^{m-2 j+1}\left(-C^{2}\right)^{j}(\xi-\tilde{x}) \quad(\text { by }(7.7)) \\
& +\sum_{j=0}^{[m / 2]} b_{m-2 j}^{m} \tilde{A}^{-n-2 m+2 j}(m-2 j) \widetilde{B}^{m-2 j-1}\left(-C^{2}\right)^{j+1}(\xi-\tilde{x}) \quad(\text { by }(7.8)) \\
& +\sum_{j=0}^{[m / 2]} b_{m-2 j}^{m} \tilde{A}^{-n-2 m+2 j} \widetilde{B}^{m-2 j}\left(-C^{2}\right)^{j} x \quad(\text { by }(7.9)) \\
& +\sum_{j=0}^{(m / 2)} b_{m-2 j-1}^{m}(n+2 m-2 j-2) \tilde{A}^{-n-2 m+2 j} \widetilde{B}^{m-2 j}\left(-C^{2}\right)^{j} x \quad(\text { by }(7.7)) \\
& +\sum_{j=0}^{(m / 2)} b_{m-2 j-1}^{m} \tilde{A}^{-n-2 m+2 j+2}(m-2 j-1) \widetilde{B}^{m-2 j-2}\left(-C^{2}\right)^{j+1} x \quad(\text { by }(7.8))
\end{aligned}
$$

Now $-C^{2}$ appears with the exponent $j+1$ in the second and the last summations, so we must replace $j$ by $j-1$ throughout in these two summations. Then relying on elementary formulas:

$$
\begin{array}{ll}
\text { (7.11) }\left[\frac{m}{2}\right]=\left[\frac{m+1}{2}\right]-1 \text { and }\left(\frac{m}{2}\right)=\left(\frac{m+1}{2}\right) \quad \text { for odd } m, \\
\text { (7.12) }\left[\frac{m}{2}\right]=\left[\frac{m+1}{2}\right] \quad \text { and }\left(\frac{m}{2}\right)=\left(\frac{m+1}{2}\right)-1 & \text { for even } m, \\
\text { (7.13) }\left[\frac{m}{2}\right]=\left(\frac{m+1}{2}\right) & \text { and }\left(\frac{m}{2}\right)=\left[\frac{m+1}{2}\right]-1 \quad \text { for all } m,
\end{array}
$$

we readjust the range of $j$ whenever necessary, for example, for odd $m$,

$$
\sum_{j=1=0}^{[m / 2]}=\sum_{j=1}^{[m / 2]+1}=\sum_{j=1}^{[(m+1) / 2]}(\text { by }(7.11)),
$$

and continue as follows. 


$$
\begin{aligned}
(\mathbf{x} \cdot \tilde{\boldsymbol{\partial}})^{m+1} g(\xi, \tilde{x}) & \\
= & \sum_{j=0}^{[m / 2]} b_{m-2 j}^{m}(n+2 m-2 j) \tilde{A}^{-n-2(m+1)+2 j} \widetilde{B}^{(m+1)-2 j}\left(-C^{2}\right)^{j}(\xi-\tilde{x}) \\
& +\sum_{j=1}^{[(m+1) / 2]} b_{m-2 j+2}^{m} \tilde{A}^{-n-2(m+1)+2 j}(m-2 j+2) \widetilde{B}^{(m+1)-2 j}\left(-C^{2}\right)^{j}(\xi-\tilde{x}) \\
& +\sum_{j=0}^{((m+1) / 2)} b_{m-2 j}^{m} \tilde{A}^{-n-2(m+1)+2 j+2} \widetilde{B}^{(m+1)-2 j-1}\left(-C^{2}\right)^{j} x \\
& +\sum_{j=0}^{(m / 2)} b_{m-2 j-1}^{m}(n+2 m-2 j-2) \tilde{A}^{-n-2(m+1)+2 j+2} \widetilde{B}^{(m+1)-2 j-1}\left(-C^{2}\right)^{j} x \\
& +\sum_{j=1}^{((m+1) / 2)} b_{m-2 j+1}^{m} \tilde{A}^{-n-2(m+1)+2 j+2}(m-2 j+1) \widetilde{B}^{(m+1)-2 j-1}\left(-C^{2}\right)^{j} x .
\end{aligned}
$$

In the second summation above, although $j$ should run from 1 to $[(m+1) / 2]+1$ for even $m$ in view of (7.12), as $j$ reaches this upper limit of summation the factor $m-2 j+2$ vanishes. And similar situations are observed for $j$ in the last summation. Now, combining the first two and the last three summations separately, we obtain

$$
\begin{aligned}
(\mathbf{x} \cdot \tilde{\boldsymbol{\partial}})^{m+1} g(\xi, \tilde{x})= & \sum_{j=0}^{[(m+1) / 2]} b_{(m+1)-2 j}^{m+1} \widetilde{A}^{-n-2(m+1)+2 j} \widetilde{B}^{(m+1)-2 j}\left(-C^{2}\right)^{j}(\xi-\tilde{x}) \\
& +\sum_{j=0}^{((m+1) / 2)} b_{(m+1)-2 j-1}^{m+1} \tilde{A}^{-n-2(m+1)+2 j+2} \widetilde{B}^{(m+1)-2 j-1}\left(-C^{2}\right)^{j} x,
\end{aligned}
$$

where

$b_{(m+1)-2 j}^{m+1}=b_{m-2 j}^{m}(n+2 m-2 j)+b_{m-2 j+2}^{m}(m-2 j+2) \quad$ for $0 \leq j \leq\left[\frac{m+1}{2}\right]$, and

$$
\begin{aligned}
b_{(m+1)-2 j-1}^{m+1}= & b_{m-2 j}^{m}+b_{m-2 j-1}^{m}(n+2 m-2 j-2) \\
& +b_{m-2 j+1}^{m}(m-2 j+1) \text { for } 0 \leq j \leq\left(\frac{m+1}{2}\right),
\end{aligned}
$$

provided that we set $b_{k}^{m}:=0$ for any $k$ not satisfying $0 \leq k \leq m$. Thus (7.5) with $m$ replaced by $m+1$ is established.

As for (7.6) with $m$ replaced by $m+1$, if we let

$$
b_{*}^{m}:=\operatorname{Max}\left\{b_{i}^{m}: 0 \leq i \leq m\right\},
$$

then

$$
\begin{aligned}
b_{(m+1)-2 j}^{m+1} & \leq b_{*}^{m}(n+2 m-2 j)+b_{*}^{m}(m-2 j+2)=b_{*}^{m}(n+3 m-4 j+2) \\
& \leq b_{*}^{m}(n+3(m+1)) \quad \text { for } 0 \leq j \leq\left[\frac{m+1}{2}\right]
\end{aligned}
$$


and

$$
\begin{aligned}
b_{(m+1)-2 j-1}^{m+1} & \leq b_{*}^{m}+b_{*}^{m}(n+2 m-2 j-2)+b_{*}^{m}(m-2 j+1) \\
& =b_{*}^{m}(n+3 m-4 j) \\
& \leq b_{*}^{m}(n+3(m+1)) \quad \text { for } 0 \leq j \leq\left(\frac{m+1}{2}\right) .
\end{aligned}
$$

Consequently, combining both inequalities, we obtain

$$
\begin{aligned}
b_{*}^{m+1} & \leq b_{*}^{m}[n+3(m+1)] \\
& \leq\left[\prod_{k=1}^{m}(n+3 k)\right][n+3(m+1)] \quad(\text { by }(7.6)) \\
& =\prod_{k=1}^{m+1}(n+3 k),
\end{aligned}
$$

and (7.6) with $m$ replaced by $m+1$ is also established.

Lemma 7.3. If $g(\xi, x)=|\xi-x|^{-n}(\xi-x)$, then for $m \geq 1$

$$
\left|(\mathbf{x} \cdot \tilde{\boldsymbol{\partial}})^{m} g(\xi, \tilde{x})\right| \leq(m+1)\left[\prod_{k=1}^{m}(n+3 k)\right]|\xi-\tilde{x}|^{-n-m+1}|x|^{m} .
$$

Proof. Referring to (7.5) and noting that $\tilde{A}=|\xi-\tilde{x}| \geq 0, C=|x| \geq 0$, and $\widetilde{B}=\mathbf{x} \cdot(\boldsymbol{\xi}-\tilde{\mathbf{x}}) \leq\|\mathbf{x}\|\|\boldsymbol{\xi}-\tilde{\mathbf{x}}\|=|x| \xi-\tilde{x} \mid=C \tilde{A}$, we obtain

$$
\begin{aligned}
\left|(\mathbf{x} \cdot \tilde{\boldsymbol{\partial}})^{m} g(\xi, \tilde{x})\right| \leq & \sum_{j=0}^{[m / 2]} b_{m-2 j}^{m} \tilde{A}^{-n-2 m+2 j}(\tilde{A} C)^{m-2 j}\left(C^{2}\right)^{j} \tilde{A} \\
& +\sum_{j=0}^{(m / 2)} b_{m-2 j-1}^{m} \tilde{A}^{-n-2 m+2 j+2}(\tilde{A} C)^{m-2 j-1}\left(C^{2}\right)^{j} C \\
= & \sum_{j=0}^{[m / 2]} b_{m-2 j}^{m} \tilde{A}^{-n-m+1} C^{m}+\sum_{j=0}^{(m / 2)} b_{m-2 j-1}^{m} \tilde{A}^{-n-m+1} C^{m} \\
\leq & (m+1)\left[\prod_{k=1}^{m}(n+3 k)\right] \tilde{A}^{-n-m+1} C^{m} \quad(\text { by }(7.6)) .
\end{aligned}
$$

Lemma 7.4. If $R_{m}(\xi, x)$ is as given in (6.3), and $|\xi|=a,|x|=r<a$, then for $m \geq 1$

$$
\left|R_{m}(\xi, x)\right| \leq(m+1)\left[\prod_{k=1}^{m}\left(1+\frac{n}{3 k}\right)\right](a-r)^{-n+1}[3 r /(a-r)]^{m} .
$$

Proof. Under $|\xi|=a,|x|=r<a$, and $\tilde{x}=x \tilde{t}, 0 \leq \tilde{t} \leq 1$, (7.14) leads to

$$
\left|(\mathbf{x} \cdot \tilde{\boldsymbol{\partial}})^{m} g(\xi, \tilde{x})\right| \leq(m+1)\left[\prod_{k=1}^{m}(n+3 k)\right](a-r)^{-n-m+1} r^{m}
$$


Using this estimate for the integral in (7.1), we obtain after integrating with respect to $\tilde{t}$

$$
\begin{aligned}
\left|R_{m}(\xi, x)\right| & \leq(m+1)\left[\prod_{k=1}^{m}(n+3 k)\right](a-r)^{-n-m+1} r^{m}(1 / m !) \\
& =(m+1)\left[\prod_{k=1}^{m}\left(\frac{n}{3 k}+1\right)\right] 3^{m} m !(a-r)^{-n+1}[r /(a-r)]^{m}(1 / m !) \\
& =(m+1)\left[\prod_{k=1}^{m}\left(1+\frac{n}{3 k}\right)\right](a-r)^{-n+1}[3 r /(a-r)]^{m} \text {. 口 }
\end{aligned}
$$

Lemma 7.5. If $\widehat{R}_{m}(x)$ is as given in (6.5), then

$$
\lim _{m \rightarrow \infty}\left|\widehat{R}_{m}(x)\right|=0
$$

uniformly for $|x|<a / 4$.

Proof. It suffices to consider only that part of (7.15) that depends on $m$, namely

$$
\begin{aligned}
a_{m} & =(m+1)\left[\prod_{k=1}^{m}\left(1+\frac{n}{3 k}\right)\right][3 r /(a-r)]^{m} \\
& =(m+1) \prod_{k=1}^{m}\left[\left(1+\frac{n}{3 k}\right)\left(\frac{3 r}{a-r}\right)\right] .
\end{aligned}
$$

Now if $|x|=r<a / 4$, then $3 r /(a-r)<1$, and

$$
\lim _{k \rightarrow \infty}\left(1+\frac{n}{3 k}\right)\left(\frac{3 r}{a-r}\right)=\frac{3 r}{a-r}<1 .
$$

Hence, if $\varepsilon$ is any fixed number between $3 r /(a-r)$ and 1 , then for sufficiently large $k>K$, say, we have

$$
\left(1+\frac{n}{3 k}\right)\left(\frac{3 r}{a-r}\right)<\varepsilon<1 .
$$

Consequently, we have for $m>K$

$$
\begin{aligned}
a_{m} & =(m+1) \prod_{k=1}^{K}\left[\left(1+\frac{n}{3 k}\right)\left(\frac{3 r}{a-r}\right)\right] \prod_{k=K+1}^{m}\left[\left(1+\frac{n}{3 k}\right)\left(\frac{3 r}{a-r}\right)\right] \\
& \leq(m+1) \prod_{k=1}^{K}\left[\left(1+\frac{n}{3 k}\right)\left(\frac{3 r}{a-r}\right)\right] \varepsilon^{m-K} .
\end{aligned}
$$

Thus, as $m$ tends to infinity, $a_{m}$ tends to 0 , and so $\left|R_{m}(\xi, x)\right|$ tends to 0 uniformly for $|x|<a / 4$ and $|\xi|=a$. Therefore, referring to (6.5), we see that $\left|\widehat{R}_{m}(x)\right|$ tends to 0 uniformly for $|x|<a / 4$ as $m$ tends to infinity.

\section{HARMONIC FUNCTIONS}

Every holomorphic function $f=\sum_{\lambda} e_{\lambda} v_{\lambda}$ is harmonic in the sense that it satisfies the Laplace equation:

$$
\Delta f=(\partial \bar{\partial}) f=\partial(\bar{\partial} f)=0 .
$$


On the other hand,

$$
\Delta f=\Delta\left(\sum_{\lambda} e_{\lambda} v_{\lambda}\right)=\sum_{\lambda} e_{\lambda} \Delta v_{\lambda}
$$

Therefore, if $f$ is holomorphic, then every one of its components $\nu_{\lambda}$ is a real harmonic function. As carriers of real harmonic functions holomorphic functions can be useful for analysis of real harmonic functions if we can show that every real harmonic function, without exception, is a component of some holomorphic function. Corollary 8.1 below shows that this is actually the case provided that the domain of the real harmonic function is simply connected and vertically traversable. A subset of $R^{n}$ is vertically traversable if for every point $\left(x_{1}, x_{2}, \ldots, x_{n}\right)$ it contains it also contains all the points $\left(x_{1}, x_{2}, \ldots, t x_{n}\right)$ for $0 \leq t \leq 1$. Clearly, a hyperball is such a set.

We begin with a basic lemma of general interest.

Lemma 8.1. Given a polycomplex holomorphic function $f$ in a vertically-traversable simply-connected domain $\Omega$ in $R^{n}$, there exists a polycomplex holomorphic function $F$ such that $\partial F=f$ in $\Omega$.

Proof. Let $f=e_{1} v_{1}+\cdots+e_{n-1} v_{n-1}+v_{n}$, then since $f$ is holomorphic, these $n$ components satisfy the equations of holomorphy (2.1) and (2.2). Now construct $F=e_{1} V_{1}+\cdots+e_{n-1} V_{n-1}+V_{n}$ by setting

$$
\begin{array}{r}
V_{i}=\int_{0}^{x_{n}} \frac{1}{2} v_{i}\left(x_{1}, \ldots, x_{n-1}, \tilde{x}_{n}\right) d \tilde{x}_{n}+\partial_{i} \phi\left(x_{1}, \ldots, x_{n-1}\right) \\
\text { for } 1 \leq i \leq n-1,
\end{array}
$$

$$
V_{n}=\int_{0}^{x_{n}} \frac{1}{2} v_{n}\left(x_{1}, \ldots, x_{n-1}, \tilde{x}_{n}\right) d \tilde{x}_{n}+\Psi\left(x_{1}, \ldots, x_{n-1}, 0\right)
$$

where $\phi\left(x_{1}, x_{2}, \ldots, x_{n-1}\right)$ is a solution of the Poisson equation

$$
\Delta_{n-1} \phi=-\frac{1}{2} v_{n}\left(x_{1}, \ldots, x_{n-1}, 0\right)
$$

(see [1]) and $\Psi\left(x_{1}, \ldots, x_{n-1}, x_{n}\right)$ is such that

$$
\partial_{i} \Psi=\frac{1}{2} v_{i}\left(x_{1}, \ldots, x_{n-1}, x_{n}\right) \text { for } 1 \leq i \leq n .
$$

Such a $\Psi$ exists because of compatibility conditions (2.2) and the simplyconnectedness of $\Omega$.

First we must check that $F$ is holomorphic, namely

$$
\partial_{1} V_{1}+\partial_{2} V_{2}+\cdots+\partial_{n} V_{n}=0
$$

$$
\partial_{i} V_{j}=\partial_{j} V_{i} \text { for } 1 \leq i \neq j \leq n
$$


To check (8.5) we work out

$$
\begin{aligned}
\sum_{i=1}^{n} \partial_{i} V_{i}= & \sum_{i=1}^{n-1}\left[\partial_{i} \int_{0}^{x_{n}} \frac{1}{2} v_{i} d \tilde{x}_{n}+\partial_{i}^{2} \phi\right]+\frac{1}{2} v_{n} \quad(\text { by }(8.1) \text { and }(8.2)) \\
= & \int_{0}^{x_{n}} \frac{1}{2}\left(-\partial_{n} v_{n}\right) d \tilde{x}_{n}+\Delta_{n-1} \phi+\frac{1}{2} v_{n} \quad(\text { by }(2.1)) \\
= & -\frac{1}{2}\left[v_{n}-v_{n}\left(x_{1}, \ldots, x_{n-1}, 0\right)\right] \\
& -\frac{1}{2} v_{n}\left(x_{1}, \ldots, x_{n-1}, 0\right)+\frac{1}{2} v_{n} \quad(\text { by }(8.3)) \\
= & 0
\end{aligned}
$$

so (8.5) is satisfied.

As for (8.6) we have for $1 \leq i \leq n-1$ and $j \neq i$

$$
\begin{aligned}
\partial_{i} V_{j} & =\partial_{i} \int_{0}^{x_{n}} \frac{1}{2} v_{j} d \tilde{x}_{n}+\partial_{i} \partial_{j} \phi \quad(\text { by }(8.1)) \\
& =\int_{0}^{x_{n}} \frac{1}{2} \partial_{j} v_{i} d \tilde{x}_{n}+\partial_{i} \partial_{j} \phi \quad(\text { by }(2.2)) \\
& =\partial_{j}\left[\int_{0}^{x_{n}} \frac{1}{2} v_{i} d \tilde{x}_{n}+\partial_{i} \phi\right]=\partial_{j} V_{i} \quad(\text { by }(8.1)) .
\end{aligned}
$$

Furthermore for $i=n$ and $j \neq n$ we have

$$
\begin{aligned}
\partial_{n} V_{j} & =\partial_{n} \int_{0}^{x_{n}} \frac{1}{2} v_{j} d \tilde{x}_{n}=\frac{1}{2} v_{j} \quad(\text { by }(8.1)) \\
\partial_{j} V_{n} & =\partial_{j} \int_{0}^{x_{n}} \frac{1}{2} v_{n} d \tilde{x}_{n}+\frac{1}{2} v_{j}\left(x_{1}, \ldots, x_{n-1}, 0\right) \quad(\text { by }(8.2) \text { and }(8.4)) \\
& =\frac{1}{2} \int_{0}^{x_{n}} \partial_{n} v_{j} d \tilde{x}_{n}+\frac{1}{2} v_{j}\left(x_{1}, \ldots, x_{n-1}, 0\right)=\frac{1}{2} v_{j} \quad(\text { by }(2.2))
\end{aligned}
$$

So we see (8.6) is satisfied.

Finally we check $\partial F=f$. Since $\bar{\partial} F=0$, we have

$$
\begin{aligned}
\partial F & =(\partial+\bar{\partial}) F=2 \partial_{n} F=2 \partial_{n}\left(\sum_{i=1}^{n} e_{i} V_{i}\right)=2 \sum_{i=1}^{n} e_{i} \partial_{n} V_{i} \\
& =2 \sum_{i=1}^{n} e_{i}\left(\frac{1}{2} v_{i}\right) \quad(\text { by }(8.1) \text { and }(8.2)) \\
& =f . \quad
\end{aligned}
$$

Using Lemma 8.1, we can show that a polycomplex harmonic function can be decomposed into a polycomplex holomorphic function and a polycomplex coholomorphic function. A hypercomplex function $g$ is coholomorphic if $\partial g=$ 0 . Obviously, $g$ is coholomorphic if and only if $\bar{g}$ is holomorphic since $\partial g=0$ and $\bar{\partial} \bar{g}=0$ are equivalent.

Theorem 8.1. In a vertically-traversable simply-connected domain $\Omega$ in $R^{n} a$ polycomplex function $h$ is harmonic if and only if

$$
h=f+g
$$


where $f$ is a polycomplex holomorphic function and $g$ is a polycomplex coholomorphic function.

Proof. First, if $h=f+g$ with $\bar{\partial} f=\partial g=0$, then

$$
\Delta h=\Delta(f+g)=(\partial \bar{\partial}) f+(\bar{\partial} \partial) g=0,
$$

so $h$ is harmonic.

Conversely, if $\Delta h=0$, then $\bar{\partial}(\partial h)=0$ so that $\partial h=f_{0}$ is a polycomplex holomorphic function. In other words, $h$ is a particular solution of the differential equation

$$
\partial u=f_{0}
$$

in which $f_{0}$ is a polycomplex holomorphic function. Therefore, it suffices to show that the general polycomplex solution of $(8.7)$ is given by

$$
u=f+g
$$

where $f$ is a polycomplex holomorphic function and $g$ is a polycomplex coholomorphic function. Now the general polycomplex solution of $(8.7)$ is routinely found by putting together the general polycomplex solution of the associated homogeneous equation

$$
\partial u=0,
$$

which is none other than the totality of polycomplex coholomorphic functions, say $\{g\}$, and a particular polycomplex solution of (8.7), say $f$, a polycomplex holomorphic function whose existence is guaranteed by Lemma 8.1.

Corollary 8.1. If a real function $h$ is harmonic in a vertically-traversable simplyconnected domain $\Omega$ in $R^{n}$, then there exists a polycomplex holomorphic function $f$ such that $h=\operatorname{Re} f$.

Proof. A real harmonic function $h$ is certainly a polycomplex harmonic function, hence by the theorem just proved

$$
h=f_{1}+g_{1}
$$

for some polycomplex $f_{1}$ and $g_{1}$ with $\bar{\partial} f_{1}=\partial g_{1}=0$. But since $h$ is real, we have

$$
h=\operatorname{Re}\left[f_{1}+g_{1}\right]=\operatorname{Re}\left[f_{1}+\bar{g}_{1}\right]=: \operatorname{Re} f
$$

with $\bar{\partial} f=\bar{\partial}\left(f_{1}+\bar{g}_{1}\right)=\bar{\partial} f_{1}+\overline{\partial g_{1}}=0$.

Thus since a real harmonic function in a suitably connected domain is the real component of a polycomplex holomorphic function, and since a holomorphic function has a Taylor series expansion, such an expansion implies a series expansion for the real harmonic function.

Theorem 8.2. If a real function $h$ is harmonic in a neighborhood of a solid sphere $\|\mathbf{x}\| \leq a$ in $R^{n}$, then it can be expanded in the following series of homogeneous harmonic polynomials.

$$
\left.h(\mathbf{x})=\sum_{|\beta|=0}^{\infty} a_{\beta} \operatorname{Re} Z^{\beta}(\mathbf{x})+b_{\beta} \operatorname{Im}_{1} Z^{\beta}(\mathbf{x}) \quad \text { (harmonic expansion in } R^{n}\right),
$$


where $\operatorname{Im}_{1} Z^{\beta}=\operatorname{comp}_{1} Z^{\beta}$ denotes the first imaginary component of the symmetric power $Z^{\beta}$, and

$$
a_{\beta}=D^{\beta} h(\mathbf{0}) /|\beta| ! \text { and } b_{\beta}=D^{\beta-e^{1}} \partial_{n} h(\mathbf{0}) /|\beta| !
$$

The convergence is uniform for $\|\mathbf{x}\| \leq a / 4$.

Proof. In view of Corollary 8.1 let $h=\operatorname{Re} f$ where $f=e_{1} v_{1}+\cdots+e_{n-1} v_{n-1}+h$ is holomorphic. Now,

$$
\begin{aligned}
\operatorname{Re} f & =\operatorname{Re}\left[\sum_{|\beta|=0}^{\infty} Z^{\beta} D^{\beta} f(\mathbf{0}) /|\beta| !\right] \quad(\text { by }(6.1)) \\
& =\sum_{|\beta|=0}^{\infty}\left[\left(\operatorname{Re} Z^{\beta}\right)\left(D^{\beta} h(\mathbf{0})\right)-\sum_{i=1}^{n-1}\left(\operatorname{comp}_{i} Z^{\beta}\right)\left(D^{\beta} v_{i}(\mathbf{0})\right)\right] /|\beta| ! .
\end{aligned}
$$

But in view of (3.3) we can rework the second summation at each $|\beta|=m \geq 0$ :

$$
\sum_{|\beta|=m} \sum_{i=1}^{n-1}\left(\operatorname{comp}_{i} Z^{\beta}\right)\left(D^{\beta} v_{i}(\mathbf{0})\right)=\sum_{|\beta|=m} \sum_{i=1}^{n-1}\left(\operatorname{comp}_{1} Z^{\beta-e^{i}+e^{1}}\right)\left(D^{\beta} v_{i}(\mathbf{0})\right) .
$$

If $\beta$ in the last double summations is replaced throughout by $\beta+e^{i}-e^{1}$, this last sum becomes

$$
\begin{aligned}
\sum_{|\beta|=m} & \sum_{i=1}^{n-1}\left(\operatorname{comp}_{1} Z^{\beta}\right)\left(D^{\beta+e^{i}-e^{1}} v_{i}(\mathbf{0})\right)=\sum_{|\beta|=m} \sum_{i=1}^{n-1}\left(\operatorname{comp}_{1} Z^{\beta}\right)\left(D^{\beta-e^{1}} \partial_{i} v_{i}(\mathbf{0})\right) \\
= & \sum_{|\beta|=m}\left(\operatorname{comp}_{1} Z^{\beta}\right) D^{\beta-e^{1}}\left(\sum_{i=1}^{n-1} \partial_{i} v_{i}(\mathbf{0})\right) \\
= & \sum_{|\beta|=m}\left(\operatorname{comp}_{1} Z^{\beta}\right) D^{\beta-e^{1}}\left(-\partial_{n} h(\mathbf{0})\right) \quad(\text { by }(2.1))
\end{aligned}
$$

Hence,

$$
h(\mathbf{x})=\sum_{|\beta|=0}^{\infty}\left[D^{\beta} h(\mathbf{0}) \operatorname{Re} Z^{\beta}+D^{\beta-e^{1}} \partial_{n} h(\mathbf{0}) \operatorname{Im}_{1} Z^{\beta}\right] /|\beta| !
$$

In particular, if $n=2,(8.9)$ reduces to

$$
h(x, y)=\sum_{m=0}^{\infty} a_{m} \operatorname{Re}(x+i y)^{m}+b_{m} \operatorname{Im}(x+i y)^{m}
$$

(harmonic expansion in $R^{2}$ ),

where

$$
a_{m}=\left(\partial^{m} / \partial x^{m}\right) h(0,0) / m ! \text { and } b_{m}=\left(\partial^{m} / \partial x^{m-1} \partial y\right) h(0,0) / m ! .
$$

This is a refinement of a result obtained in [8].

As an immediate consequence of the expansion formula (8.9) we can enumerate the number of independent homogeneous harmonic polynomials of degree $m$ for each $m \geq 0$. 
Corollary 8.2. In $R^{n}$ for each $m \geq 0$ there are exactly

$$
N(m, n)=(m+n-3) !(2 m+n-2) / m !(n-2) !
$$

independent homogeneous harmonic polynomials of degree $m$.

Proof. Polynomials of degree $m$ in (8.9) are $\operatorname{Re} Z^{\beta}$ and $\operatorname{Im}_{1} Z^{\beta}$ with $|\beta|=$ $m$. Although each $Z^{\beta}$ has a real component, only $Z^{\beta}$ with $\beta_{1} \geq 1$ has a first imaginary component. Hence we need only determine the cardinalities of the sets $\left\{Z^{\beta}:|\beta|=m\right\}$ and $\left\{Z^{\beta}:|\beta|=m, \beta_{1} \geq 1\right\}$. Now obviously the cardinality of the first set is no different from the number of ways in which $m$ identical objects can be distributed among $k=n-1$ distinct individuals, which is given by a well-known combinatorial formula (see [3] for example) as

$$
C_{m}^{m+k-1}:=(m+k-1) ! / m !(k-1) !=(m+n-2) ! / m !(n-2) !=: C_{m}^{m+n-2}
$$

As for the cardinality of the second set

$$
\left\{Z^{\beta}:|\beta|=m, \beta_{1} \geq 1\right\}=\left\{Z^{\beta}:|\beta|=m\right\}-\left\{Z^{\beta}:|\beta|=m, \beta_{1}=0\right\},
$$

we have

$$
C_{m}^{m+n-2}-C_{m}^{m+n-3}=(m+n-2) ! / m !(n-2) !-(m+n-3) ! / m !(n-3) ! .
$$

Hence combining (8.13) and (8.14) we have altogether

$$
2(m+n-2) ! / m !(n-2) !-(m+n-3) ! / m !(n-3) !=(m+n-3) ! / m !(n-2) !
$$

In particular, $N(m, 3)=2 m+1$, and $N(m, 2)=2$. In fact, for $R^{2}$ the two independent homogeneous harmonic polynomials of degree $m$ are just the real and the imaginary components of the power $z^{m}=(x+i y)^{m}$ as shown in (8.11).

\section{REFERENCES}

1. R. Courant and D. Hilbert, Methods of mathematical physics, Vol. 2, Wiley-Interscience, New York, 1962.

2. R. Delanghe, On regular analytic functions with values in Clifford algebra, Math. Ann. 185 (1970), 91-111.

3. W. Feller, An introduction to probability theory and its applications, Vol. 1, Wiley, New York, 1968.

4. W. K. Hayman, Power series expansions for harmonic functions, Bull. London Math. Soc. 2 (1970), 152-158.

5. G. N. Hile, Representations of solutions of a special class of first order systems, J. Differential Equations 25 (1977), 410-424.

6. G. N. Hile and P. Lounesto, Matrix representations of Clifford algebra, Linear Algebra Appl. 128 (1990), 51-63.

7. R. Z. Yeh, Hyperholomorphic junctions and higher order partial differential equations in the plane, Pacific J. Math. 142 (1990), 379-399.

8. Solutions of polyharmonic Dirichlet problems derived from general solutions in the plane, J. Math. Anal. Appl. 154 (1991), 341-363.

9. Hyperholomorphic functions and second order partial differential equations in $R^{n}$, Trans. Amer. Math. Soc. 325 (1991), 287-318. 